\title{
Disentangling sources of interference in the $N$-back task
}

\author{
Long $\mathrm{Ni}^{1}{ }^{*}$ Wei Ji Ma ${ }^{1,2}$ \\ ${ }^{1}$ Center for Neural Science and ${ }^{2}$ Department of Psychology, New York University
}

\begin{abstract}
The $N$-back task is a common working memory paradigm: subjects judge whether the current item in a sequence (the probe) matches the one presented $N$ items ago (the target). Performance in the $N$-back task is susceptible to interference from intervening items (distractors), especially when the probe and the distractor are similar. However, the categorical stimuli usually used in the classic $N$-back task are not suitable for distinguishing possible causes of interference. Here, we instead used parametric stimuli (varying in orientation or color) to measure the amount of interference induced in a 2-back task by the 1-back distractor. As expected, we find that interference was larger when the feature distance between the probe and the distractor was smaller. To investigate the source of the interference, we fitted four mathematical models to the data. In the No interference model, the observer utilizes an optimal decision strategy. In the Early pooling model, the observer has access only to a mixed measurement of the target and distractor, but employs an optimal de-mixing strategy. In the Late pooling model, the observer mixes decision variables that are optimal for the target and distractor separately. In the Substitution model, the observer swaps the target with the distractor on some proportion of trials, and decides optimally otherwise. The No interference and the Early pooling models described the data substantially worse than the Late pooling and the Substitution models. However, we were not able to distinguish the Late pooling and Substitution models. Our modeling results suggest that interference in the 2-back task results either from the pooling of decision variables based the "target" and "distractor" separately, or from the substitution of the distractor for the target. Our study opens the door to parametrically examining the sources of interference in similar cognitive tasks.
\end{abstract}

*present address: Department of Psychology, University of Pennsylvania, Philadelphia, PA 19104 
Keywords: $N$-back task, interference, pooling, substitution, psychophysics, behavioral modeling

\section{Introduction}

The $N$-back task is a classic paradigm for studying the capacity of working memory. It requires a participant to judge whether the current stimulus in a sequence matches the one that is presented $N$ stimuli earlier in the sequence. In order to perform the task, the participant needs to memorize, maintain, and update the current item (probe), the $N$-back item (target) as well as the intervening item or items (distractors) in a continuous manner (Kirchner, 1958; Jaeggi et al., 2010). As the sequence unfolds, a given stimulus will start out as the probe, then become a distractor (unless $N=1$ ), then the target, before finally becoming irrelevant.

Although the $N$-back task is widely used, the mechanisms of interference due to the distractor are not known. Previous studies have shown that the distractor induces greater interference when it resembles the probe. Taking the 2-back task as an example, a 1-back distractor (lure) that was the same as the probe (e.g., A-B-B) elicited more false alarms and misses than a 1-back distractor that was different from the probe (e.g., A-B-C) (Kane et al., 2007; Moore and Ross, 1963). The effect of target-distractor similarity on the extent of interference has also been revealed in other working memory tasks, such as verbal and spatial serial order memory tasks (Oberauer et al., 2004; Awh et al., 2007). Performance on these tasks is impaired when the target and distractor are from the same category (e.g., both are letters) (Conlin and Gathercole, 2006) or when target-distractor feature similarity within the same category is high (Oberauer et al., 2004; Awh et al., 2007).

Interference in the $N$-back task has an interesting and underappreciated parallel in visual crowding, the phenomenon that stimuli that flank a target impair an observer's ability to recognize the target (see Levi, 2008, for a review). The flanking stimuli can be viewed as analogous to the distractor(s) in the $N$-back task. Indeed, the magnitude of visual crowding depends strongly on target-distractor similarity and is stronger when the features of the target and distractors are similar (e.g., Põder, 2007; Chung and Mansfield, 2009; Bernard and Chung, 2011).

To quantitatively measure interference as a function of target-distractor similarity in crowding, researchers often employ parametric stimuli such as position (Greenwood et al., 2009) or orientation (Ester et al., 2014, 2015). By contrast, in the vast majority of previous $N$-back studies, the stimuli were non-parametric, such as letters and numbers (e.g., Jaeggi et al., 2010; Pochon et al., 2002; Kane et al., 2007; Oberauer, 2005). This makes quantitatively characterizing the magnitude of 
similarity-based interference from the distractor difficult. In the current study, our first goal is to characterize how target-distractor similarity affects the magnitude of distractor-induced interference in the 2-back task.

In the visual crowding literature, two broad computational hypotheses exist. The first hypothesis is that crowding results from the pooling or integration of features of the target and flankers (Parkes et al., 2001; Greenwood et al., 2009; Dakin et al., 2010). The observer's report of a target feature is then dependent on a weighted average of the target and distractor features. The second hypothesis is that crowding is caused by the observer's confusion between the target and flankers (Ester et al., 2014, 2015; Nandy and Tjan, 2007). The observer would swap the target and one of the distractors on some proportion of trials. Thus, their report is based on the feature of either the target or one of the flankers on a single trial. By using parametric stimuli and comparing models, progress has been made in distinguishing between the pooling and substitution hypotheses (Freeman et al., 2012; Keshvari and Rosenholtz, 2016; Harrison and Bex, 2017).

Inspired by these hypotheses in the field of visual crowding, we here consider two possible sources of interference induced by the 1-back distractor in the 2-back task. Specifically, we propose that interference in the 2-back task could result from the observer either a) substituting the distractor for the target (substitution hypothesis), i.e., they lose track of which one is target and which one is distractor or b) mixing aspects of the target and distractor (pooling hypothesis). Within the pooling hypothesis, we consider two variants: one model in which the observer mixes the features of the target and distractor, as in the model for crowding (Early pooling), and a new model in which the observer mixes the strengths of evidence (as measured by a continuous decision variable) based on the target and distractor separately (Late pooling). Thus, our second goal is to distinguish the different possible sources of interference in the 2-back task. We aimed to achieve this by comparing two pooling models (Early vs. Late pooling), a substitution model, and an optimal model that assumes no interference from the 1-back distractor.

\section{Models}

\section{Brief description of tasks}

In order to measure interference psychophysically, we created a variant of the classic 2-back task, which we call the Parametric Two-Back Task. The relevant stimulus feature was either orientation or color. The feature took values on a continuous, circular feature space. Across the stimuli in a sequence, the feature value varied 
(Fig.1). The subject was asked to continuously compare the feature value of the stimulus they just saw (the probe) with the one 2 stimuli ago in the sequence (the 2back stimulus or the target). To do well on the task, the subject also had to memorize the feature value of the 1-back stimulus (the distractor), as it would become the target next. Therefore, the 1-back stimulus might induce interference in working memory and consequently affect performance. The parametric manipulation of the stimulus feature allows us to characterize the effects of distractor-probe similarity on performance.

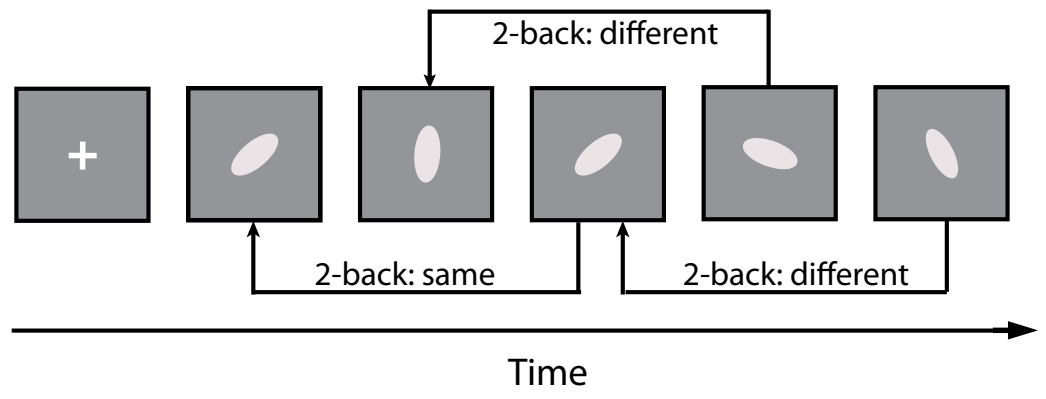

Figure 1: Schematic of the Parametric Two-Back Task. The task uses a parametric stimulus feature, here orientation. The subject starts by fixating and then views a sequence of oriented ellipses. For each ellipse in the sequence (the probe), the subject reports through a key press whether or not its orientation matches the orientation of the ellipse 2 back in the sequence (the target). The 1-back ellipse, which is the distractor on the current trial, becomes the target on the next trial. Thus, the observer cannot afford to ignore the 1-back distractor.

\section{Brief description of models}

We use computational modeling to distinguish different possible mechanisms underlying interference in our 2-back task. We tested four models on our data (Fig. 2). All models consist of an encoding stage and a decision stage. In all models, the core of the encoding stage is the representation of target, distractor, and probe as noisy measurements, and the core of the decision stage is an optimal observer acting on those measurements. The No-Interference (NI) model postulates that the distractor does not play a role in either the encoding or the decision stage. In two other models, the distractor gets "mixed" with the target; we call these models pooling models by analogy to similar models for crowding (Parkes et al., 2001; Greenwood et al., 2009). The locus of mixing in the Early Pooling (EP) model is the encoding stage, and in the Late Pooling (LP) model the decision stage. Finally, we tested a Substitution 
(Sub) model, which asserts that interference results from the observer confusing the identities of the target and distractor with some probability.

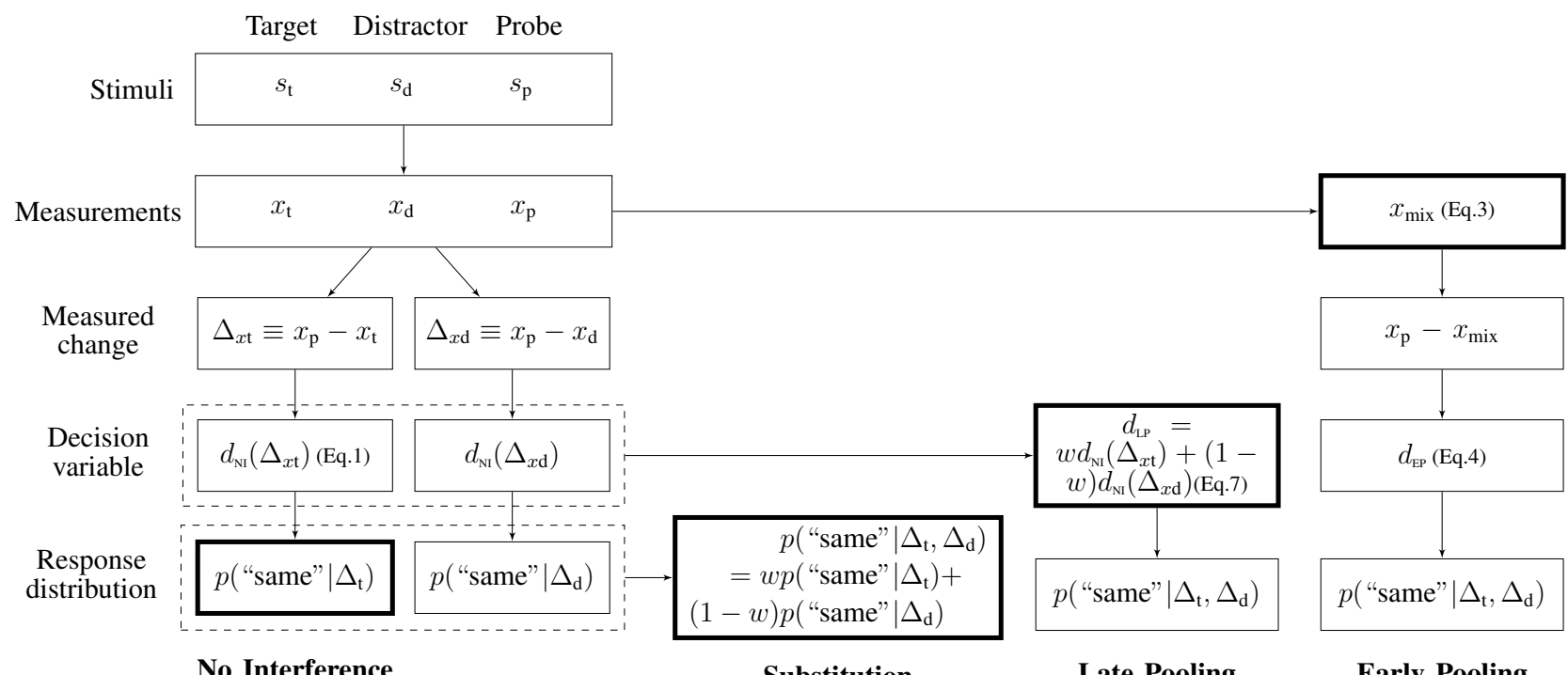

Figure 2: Four models of behavior in the Parametric Two-Back Task. In all models, the target (2-back stimulus), distractor (1-back stimulus), and probe (0-back stimulus) are internally represented as noisy measurements $x_{\mathrm{t}}, x_{\mathrm{d}}$, and $x_{\mathrm{p}}$, respectively. In the $N_{o}$ Interference (NI) model, the observer computes an optimal decision variable, $d_{\mathrm{NI}}$, based on the circular difference between the measurements of the probe and the target, $\Delta_{x \mathrm{t}}$. The circular difference between the measurements of the probe and the distractor, $\Delta_{x \mathrm{~d}}$, does not play a role. In the Early Pooling (EP) model, the measurements of the target and distractor get mixed; $x_{\text {mix }}$ is the weighted circular mean of $x_{\mathrm{t}}$ and $x_{\mathrm{d}}$. In the Late Pooling (LP) model, the optimal decision variables based on the target and the distractor get mixed. In both pooling models, $w$ is the weight assigned to the target. In the Substitution model, the observer swaps the target for the distractor with probability $1-w$, thus basing their response on either $\Delta_{x \mathrm{t}}$ or $\Delta_{x \mathrm{~d}}$. $\Delta_{\mathrm{t}}$ indicates the circular difference between the probe and target $\left(s_{\mathrm{p}}-s_{\mathrm{t}}\right)$ and $\Delta_{\mathrm{d}}$ is the circular difference between the probe and distractor $\left(s_{\mathrm{p}}-s_{\mathrm{d}}\right)$.

\section{No interference model}

In the No Interference (NI) model, the 1-back distractor does not exert any influence on the observer's response. We now describe the encoding and decision stages of this model. 


\section{Encoding stage}

We denote the target by $s_{\mathrm{t}}$ and the probe by $s_{\mathrm{p}}$. We further denote the observer's noisy measurements of these stimuli by $x_{\mathrm{t}}$ and $x_{\mathrm{p}}$, respectively. We assume that these measurements follow Von Mises distributions with means $s_{\mathrm{t}}$ and $s_{\mathrm{p}}$, respectively, and a shared concentration parameter $\kappa$ :

$$
\begin{aligned}
& p\left(x_{\mathrm{p}} \mid s_{\mathrm{p}} ; \kappa\right)=\frac{1}{2 \pi I_{0}(\kappa)} e^{\kappa \cos \left(x_{\mathrm{p}}-s_{\mathrm{p}}\right)} \\
& p\left(x_{\mathrm{t}} \mid s_{\mathrm{t}} ; \kappa\right)=\frac{1}{2 \pi I_{0}(\kappa)} e^{\kappa \cos \left(x_{\mathrm{t}}-s_{\mathrm{t}}\right)}
\end{aligned}
$$

where $I_{0}$ is the modified Bessel function of the first kind of order 0 . The orientation of our stimuli has a natural range of $\left[-90^{\circ}, 90^{\circ}\right]$. However, in the models (i.e., equations and corresponding simulations), we remap the orientations to the range $\left[-180^{\circ}\right.$, $180^{\circ}$ ] on which the von Mises distribution is defined. In all figures, we use the true orientation space, mapping back if needed.

\section{Decision stage}

Based on the noisy measurements of the probe and target, the observer has to decide whether or not the probe matches the target. We denote "same" by $C=1$ and "different" by $C=0$. The optimal way to make this same/different decision is to choose the $C$ with the highest posterior probability. Thus, the optimal observer makes a decision based on the probabilities $p\left(C=1 \mid x_{\mathrm{p}}, x_{\mathrm{t}}\right)$ and $p\left(C=0 \mid x_{\mathrm{p}}, x_{\mathrm{t}}\right)$. We can define the decision variable as the $\log$ posterior ratio, denoted by $d_{\mathrm{NI}}$, and apply Bayes' rule:

$$
\begin{aligned}
d_{\mathrm{NI}} & =\log \frac{p\left(C=1 \mid x_{\mathrm{p}}, x_{\mathrm{t}}\right)}{p\left(C=0 \mid x_{\mathrm{p}}, x_{\mathrm{t}}\right)} \\
& =\log \frac{p(C=1)}{p(C=0)}+\log \frac{p\left(x_{\mathrm{p}}, x_{\mathrm{t}} \mid C=1\right)}{p\left(x_{\mathrm{p}}, x_{\mathrm{t}} \mid C=0\right)}
\end{aligned}
$$

where $p(C=1)$ is the observer's prior probability that the probe matches the target. The optimal observer responds "same" when the posterior of $p\left(C=1 \mid x_{\mathrm{p}}, x_{\mathrm{t}}\right)$ is greater than 0.5 , or equivalently, when $d_{\mathrm{NI}}>0$. To compute the class likelihoods 
$p\left(x_{\mathrm{p}}, x_{\mathrm{t}} \mid C=0\right)$ and $p\left(x_{\mathrm{p}}, x_{\mathrm{t}} \mid C=1\right)$, the observer needs to marginalize (average) over the hidden variables $s_{\mathrm{p}}$ and $s_{\mathrm{t}}$. Thus,

$$
d_{\mathrm{NI}}=\log \frac{p(C=1)}{p(C=0)}+\log \frac{\iint p\left(x_{\mathrm{p}} \mid s_{\mathrm{p}}\right) p\left(x_{\mathrm{t}} \mid s_{\mathrm{t}}\right) p\left(s_{\mathrm{p}}, s_{\mathrm{t}} \mid C=1\right) d s_{\mathrm{p}} d s_{\mathrm{t}}}{\iint p\left(x_{\mathrm{p}} \mid s_{\mathrm{p}}\right) p\left(x_{\mathrm{t}} \mid s_{\mathrm{t}}\right) p\left(s_{\mathrm{p}}, s_{\mathrm{t}} \mid C=0\right) d s_{\mathrm{p}} d s_{\mathrm{t}}}
$$

Substituting the distributions from the encoding model and evaluating the integrals, we find (see Appendix A for derivation):

$$
d_{\mathrm{NI}}=\log \frac{p(C=1)}{p(C=0)}+\log \frac{I_{0}\left(\kappa \sqrt{2+2 \cos \Delta_{x t}}\right)}{I_{0}(\kappa)^{2}}
$$

As expected based on symmetry considerations, the decision variable is solely determined by the difference $\Delta_{x \mathrm{t}} \equiv x_{\mathrm{p}}-x_{\mathrm{t}}$, and not by $x_{\mathrm{p}}$ and $x_{\mathrm{t}}$ individually.

\section{Response probabilities}

Our goal is to predict the probability that the observer reports "same" for a given stimulus combination $\left(s_{\mathrm{p}}, s_{\mathrm{t}}\right)$, which we denote as $p\left(\hat{C}=1 \mid s_{\mathrm{p}}, s_{\mathrm{t}}\right)$. On a single trial, the measurements $x_{\mathrm{p}}$ and $x_{\mathrm{t}}$ are not known to the experimenter. Thus, the experimenter needs to average over all possible values of $x_{\mathrm{p}}$ and $x_{\mathrm{t}}$ for a given stimulus combination $\left(s_{\mathrm{p}}, s_{\mathrm{t}}\right)$ to calculate the response probabilities on the trial in question. (This marginalization is conceptually very different from the one in "Decision stage". In the decision stage, the observer performs the marginalization over the hidden variables $s_{\mathrm{p}}$ and $s_{\mathrm{t}}$, which are unknown to the observer but known to the experimenter. Here, the experimenter marginalizes over the measurements, which are known to the observer but unknown to the experimenter.) The probability of reporting "same" for a given combination of $s_{\mathrm{p}}$ and $s_{\mathrm{t}}$ is given by:

$$
\begin{aligned}
p\left(\hat{C}=1 \mid s_{\mathrm{p}}, s_{\mathrm{t}}\right) & =\iint p\left(\hat{C}=1 \mid x_{\mathrm{p}}, x_{\mathrm{t}}\right) p\left(x_{\mathrm{p}} \mid s_{\mathrm{p}}\right) p\left(x_{\mathrm{t}} \mid s_{\mathrm{t}}\right) d x_{\mathrm{p}} d x_{\mathrm{t}} \\
& =\iint_{\left(x_{\mathrm{t}}, x_{\mathrm{p}}\right): d_{\mathrm{NI}}>0} p\left(x_{\mathrm{p}} \mid s_{\mathrm{p}}\right) p\left(x_{\mathrm{t}} \mid s_{\mathrm{t}}\right) d x_{\mathrm{p}} d x_{\mathrm{t}}
\end{aligned}
$$

where $d_{\mathrm{NI}}>0$ defines an area in the two-dimensional space spanned by $x_{\mathrm{t}}$ and $x_{\mathrm{p}}$. To calculate the response probability, we first computed the joint probability of $p\left(x_{\mathrm{p}} \mid s_{\mathrm{p}}\right) p\left(x_{\mathrm{t}} \mid s_{\mathrm{t}}\right)$ and then multipled it by the mask defined by the area $d_{\mathrm{NI}}>0$. We evaluated the resulting integral numerically on grid.

As one would expect, the result depends only on $\Delta_{\mathrm{t}} \equiv s_{\mathrm{p}}-s_{\mathrm{t}}$, but not on $s_{\mathrm{p}}$ and $s_{\mathrm{t}}$

individually (see Appendix B for derivation). Therefore, we will write $p\left(\hat{C}=1 \mid \Delta_{\mathrm{t}}\right)$ instead of $p\left(\hat{C}=1 \mid s_{\mathrm{p}}, s_{\mathrm{t}}\right)$. 


\section{Lapse rate}

Due to lapses of attention, the observer might respond by randomly guessing on a small proportion of trials. We take into account this possibility by including a free parameter of lapse rate in the NI model as well as in the following three interference models. Thus, on each trial, there is a probability $\lambda$ that the observer generates a response purely based on a random guess instead of on the decision rule of the respective model. The predicted probability of reporting "same" thus becomes:

$$
p\left(\hat{C}=1 \mid s_{\mathrm{p}}, s_{\mathrm{t}}\right)=\frac{\lambda}{2}+(1-\lambda) \iint_{\left(x_{\mathrm{t}}, x_{\mathrm{p}}\right): d_{\mathrm{NI}}>0} p\left(x_{\mathrm{p}} \mid s_{\mathrm{p}}\right) p\left(x_{\mathrm{t}} \mid s_{\mathrm{t}}\right) d x_{\mathrm{p}} d x_{\mathrm{t}}
$$

\section{Early pooling model}

The Early pooling (EP) model postulates that the 1-back distractor plays a role in the observer's responses by inducing interference at an early stage.

\section{Encoding stage}

The EP model postulates that the measurement of the target, $x_{t}$, gets mixed with the measurement of the distractor, $x_{\mathrm{d}}$. To specify this mixing, we introduce the twodimensional unit vectors corresponding to these measurements, which we denote by $\mathbf{X}_{\mathrm{t}}$ and $\mathbf{X}_{\mathrm{d}}$, respectively. The mixed measurement is associated with the weighted average of these two unit vectors (Fig. 3A):

$$
\mathbf{X}_{\text {mix }}=w \mathbf{X}_{\mathrm{t}}+(1-w) \mathbf{X}_{\mathrm{d}}
$$

As a result, the direction $x_{\text {mix }}$ of this mixed measurement vector is the weighted circular mean of $x_{\mathrm{t}}$ and $x_{\mathrm{d}}$ :

$$
x_{\text {mix }}=\operatorname{atan} 2\left(w \sin x_{\mathrm{t}}+(1-w) \sin x_{\mathrm{d}}, w \cos x_{\mathrm{t}}+(1-w) \cos x_{\mathrm{d}}\right) .
$$

$x_{\mathrm{d}}$ also follows the Von Mises distribution with a mean $s_{\mathrm{d}}$ and a shared concentration parameter $\kappa$ with $x_{\mathrm{p}}$ and $x_{\mathrm{t}}$.

\section{Decision stage}

As in the NI model, the observer makes the same/different decision based on $p(C=$ $\left.1 \mid x_{\mathrm{p}}, x_{\text {mix }}\right)$ and $p\left(C=0 \mid x_{\mathrm{p}}, x_{\text {mix }}\right)$, and chooses $C$ with the highest posterior probability. However, the probabilities are now based on the input $x_{\mathrm{p}}$ and $x_{\text {mix }}$ rather than 
$x_{\mathrm{p}}$ and $x_{\mathrm{t}}$. Applying Bayes' rule, we define the decision variable as a log posterior ratio:

$$
d_{\mathrm{EP}}=\log \frac{p\left(C=1 \mid x_{\mathrm{p}}, x_{\mathrm{mix}}\right)}{p\left(C=0 \mid x_{\mathrm{p}}, x_{\text {mix }}\right)}=\log \frac{p(C=1)}{p(C=0)}+\log \frac{p\left(x_{\mathrm{p}}, x_{\mathrm{mix}} \mid C=1\right)}{p\left(x_{\mathrm{p}}, x_{\mathrm{mix}} \mid C=0\right)}
$$

The observer reports "same" when the posterior of $p\left(C=1 \mid x_{\mathrm{p}}, x_{\text {mix }}\right)$ is greater than 0.5 , or equivalently, when $d_{\mathrm{EP}}>0$. To compute $d_{\mathrm{EP}}$, the observer needs to compute the class likelihoods $p\left(x_{\mathrm{p}}, x_{\text {mix }} \mid C=0\right)$ and $p\left(x_{\mathrm{p}}, x_{\text {mix }} \mid C=1\right)$. To do so, like in the NI model, the observer needs to marginalize over the hidden variables $s_{\mathrm{p}}$ and $s_{\mathrm{t}}$ which are unknown to them. In addition, the observer should marginalize over the hidden variable $s_{\mathrm{d}}$ as its measurement $x_{\mathrm{d}}$ is also a constituent of $x_{\text {mix }}$. Importantly, because the "pure" measurements of $x_{\mathrm{t}}$ and $x_{\mathrm{d}}$ that determine $x_{\text {mix }}$ are unknown to the observer, the observer needs to marginalize over all possible combinations of $x_{\mathrm{t}}$ and $x_{\mathrm{d}}$ that generate $x_{\mathrm{mix}}$. Thus, to compute $d_{\mathrm{EP}}$, the optimal observer would perform a marginalization over five variables: $s_{\mathrm{p}}, s_{\mathrm{t}}, s_{\mathrm{d}}, x_{\mathrm{t}}$ and $x_{\mathrm{d}}$ :

$$
\begin{aligned}
d_{\mathrm{EP}}= & \log \frac{p(C=1)}{p(C=0)}+ \\
& \log \frac{\iiint \iint p\left(x_{\mathrm{mix}}, x_{\mathrm{p}} \mid x_{\mathrm{t}}, x_{\mathrm{d}}, s_{\mathrm{p}}, s_{\mathrm{t}}, s_{\mathrm{d}}\right) p\left(x_{\mathrm{t}}, x_{\mathrm{d}}, s_{\mathrm{t}}, s_{\mathrm{d}}, s_{\mathrm{p}} \mid C=1\right) d x_{\mathrm{t}} d x_{\mathrm{d}} d s_{\mathrm{t}} d s_{\mathrm{d}} d s_{\mathrm{p}}}{\iiint \int p\left(x_{\mathrm{mix}}, x_{\mathrm{p}} \mid x_{\mathrm{t}}, x_{\mathrm{d}}, s_{\mathrm{p}}, s_{\mathrm{t}}, s_{\mathrm{d}}\right) p\left(x_{\mathrm{t}}, x_{\mathrm{d}}, s_{\mathrm{t}}, s_{\mathrm{d}}, s_{\mathrm{p}} \mid C=0\right) d x_{\mathrm{t}} d x_{\mathrm{d}} d s_{\mathrm{t}} d s_{\mathrm{d}} d s_{\mathrm{p}}}
\end{aligned}
$$

The posterior ratio can be evaluated to (see Appendix C for derivation):

$$
\begin{aligned}
& d_{\mathrm{EP}}= \\
& \left\{\begin{array}{lr}
0 & \text { if } w<0.5 \\
\log \frac{p(C=1)}{p(C=0)}+\log \frac{1}{I_{0}(\kappa)^{2}}+ & \text { if } w \geq 0.5 \\
+\log \frac{1}{2 \arcsin \frac{1-w}{w}} \int_{-\arcsin \left(\frac{1-w}{w}\right)}^{\arcsin \left(\frac{1-w}{w}\right)} I_{0}\left(\kappa \sqrt{2+2 \cos \left(x_{\mathrm{p}}-x_{\mathrm{mix}}-\tilde{x}\right)}\right) d \tilde{x} &
\end{array}\right.
\end{aligned}
$$

where $\tilde{x}$ is the difference between the hypothesized $x_{\mathrm{t}}$ and $x_{\mathrm{d}}$ and within the range of $\left[-\arcsin \left(\frac{1-w}{w}\right), \arcsin \left(\frac{1-w}{w}\right)\right]$. It should be noted that the observer is still able to make the best possible decision based on the compromised information by de-mixing all possible combinations of $x_{\mathrm{t}}$ and $x_{\mathrm{d}}$ that generate $x_{\text {mix }}$. Therefore, though the "pure" measurements of $x_{\mathrm{t}}$ and $x_{\mathrm{d}}$ that produce $x_{\text {mix }}$ are unknown to the observer, the observer needs to marginalize over only the possible combinations of $x_{\mathrm{t}}$ and $x_{\mathrm{d}}$ 
for both of which are constrained by a given $x_{\text {mix }}$. The values of $x_{\mathrm{t}}$ and $x_{\mathrm{d}}$ for a given $x_{\text {mix }}$ is constrained by (see Fig. 3B):

$$
x_{\mathrm{d}}=\operatorname{atan} 2\left(R \sin x_{\text {mix }}-w \sin x_{\mathrm{t}}, \quad R \cos x_{\text {mix }}-w \cos x_{\mathrm{t}}\right) .
$$

where $R=\sqrt{2 w^{2}-2 w+1+2 w(1-w) \cos \left(x_{\mathrm{t}}-x_{\mathrm{d}}\right)}$.

For a given $x_{\text {mix }}, x_{\mathrm{t}}$ is constrained within a certain range that varies as a function of $w$. Thus, $x_{\text {mix }}$ determines the allowable range of $x_{\mathrm{t}}$ for a fixed $w$ (Fig. 3C). Specifically, when $w<0.5$, there is no restriction on $x_{\mathrm{t}}$ (i.e., $x_{\mathrm{t}}$ could be any value from $-180^{\circ}$ to $180^{\circ}$ ), but as $w$ increases over 0.5 , the possible range of $x_{\mathrm{t}}$ is narrowing down. $x_{\mathrm{t}}$ becomes the same as $x_{\text {mix }}$ when $w$ equals 1 , indicating no mixing of $x_{\mathrm{t}}$ and $x_{\mathrm{d}}$. In this special case, the decision variable reduces to Eq. 1 in the NI model. As a result, for the decision variable $d_{\mathrm{EP}}$ we end up with two equations corresponding to the cases of $w<0.5$ and $w \geq 0.5$, respectively.

While it is true that the observer has access only to $x_{\mathrm{p}}$ and $x_{\text {mix }}$, as $x_{\text {mix }}$ is the weighted circular mean of $x_{\mathrm{t}}$ and $x_{\mathrm{d}}$, mathematically we can rewrite the decision variable in terms of $x_{\mathrm{p}}, x_{\mathrm{d}}$ and $x_{\mathrm{t}}$ (see Appendix $\mathrm{C}$ for derivation):

$$
\begin{aligned}
& d_{\mathrm{EP}}= \\
& \left\{\begin{array}{l}
0 \\
10 \\
+
\end{array}\right. \\
& \log \frac{p(C=1)}{p(C=0)}+\log \frac{1}{I_{0}(\kappa)^{2}} \\
& +\log \frac{\int_{-\arcsin \frac{1-w}{w}}^{\arcsin \frac{1-w}{w}} I_{0}\left(\kappa \sqrt{\left.2+\frac{2\left(w \cos \left(\Delta_{x \mathrm{t}}-\tilde{x}\right)+(1-w) \cos \left(\Delta_{x \mathrm{~d}}-\tilde{x}\right)\right)}{\sqrt{2 w^{2}-2 w+1+2 w(1-w) \cos \left(\Delta_{x \mathrm{t}}-\Delta_{x \mathrm{~d}}\right)}}\right)}\right.}{2 \arcsin \frac{1-w}{w}} \quad \text { if } w \geq 0.5
\end{aligned}
$$

As expected, the decision variable is determined by $\Delta_{x \mathrm{t}}$ and $\Delta_{x \mathrm{~d}}$, but not by $x_{\mathrm{p}}, x_{\mathrm{d}}$ and $x_{\mathrm{t}}$ individually. 
A

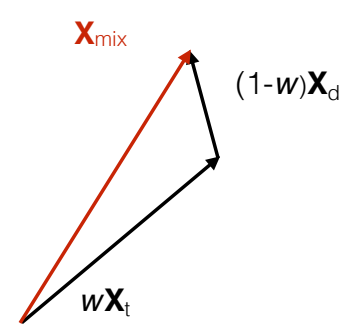

$\mathrm{D}$

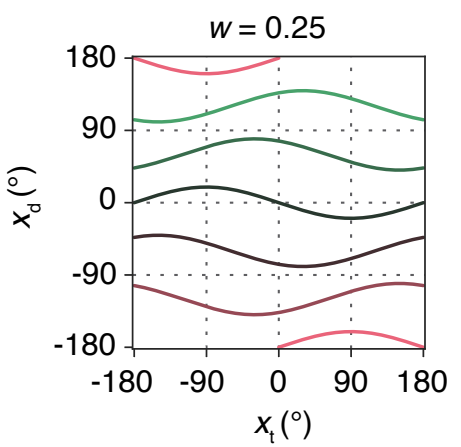

B

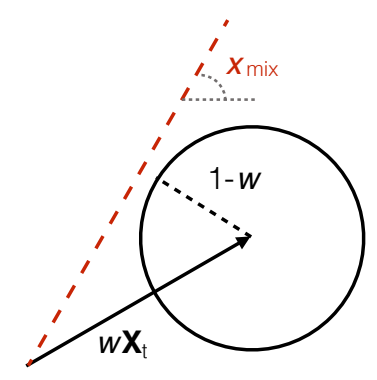

C

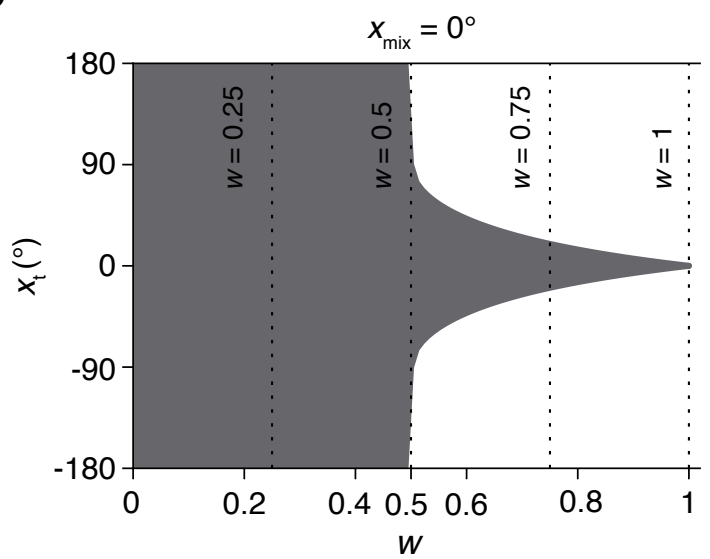

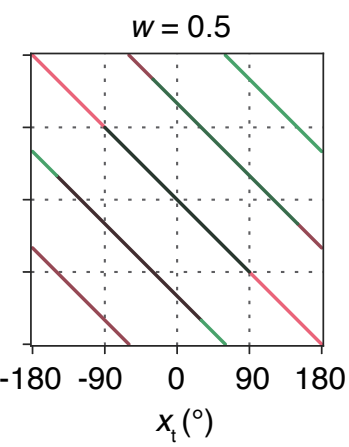
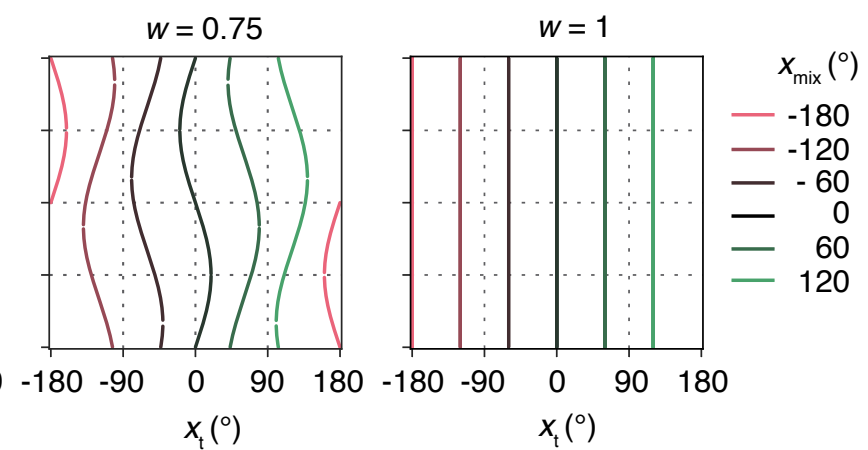

Figure 3: Under the EP model, the observer can employ an optimal decision strategy by reconstructing $x_{\mathrm{t}}$ and $x_{\mathrm{d}}$ from the compromised information $x_{\text {mix }}$. (A) The observer has access only to $X_{\text {mix }}$, which is the weighted circular mean of $X_{\mathrm{t}}$ and $X_{\mathrm{d}} \cdot X_{\mathrm{t}}$ and $X_{\mathrm{d}}$ are angle vectors with unit length. $w$ and $1-w$ are the weights of $X_{\mathrm{t}}$ and $X_{\mathrm{d}}$, respectively. (B) Constraint of $x_{\text {mix }}$ on $X_{\mathrm{t}}$ for a fixed $w$. Given a specific $w$, there is not always a possible combination of $X_{\mathrm{t}}$ and $X_{\mathrm{d}}$ that generates the $x_{\text {mix }}$ (C) All possible $x_{\mathrm{t}}$ values as a function of $w$ given $x_{\text {mix }}=0^{\circ}$. (D) De-mixing of $X_{\mathrm{t}}$ and $X_{\mathrm{d}}$ from a given $x_{\text {mix }}$ (indicated by different colored lines) for different values of $w$.

De-mixing process: an analogy with numbers. To help readers understand the de-mixing process, we make an analogy here with numbers. Consider the case where we have a number $c$, which is the weighted average of two separate numbers $(a, b)$ taken from 0 to 100 . The purpose of the de-mixing process is to find all possible values of $a$ and $b$ that could generate the specific mixed number $c$. We are able to do the de-mixing because for a given $c$, the possible values of $a$ are constrained to a certain range that varies as a function of $w$ (i.e., the weight for $a$ ). For example, for $c=50$ and $w=0.5, a$ (or $b$ ) can be any number between 0 and 100. As $w$ increases 
(or decreases), the range of possible $a$ (or b) shrinks gradually (Fig 4).
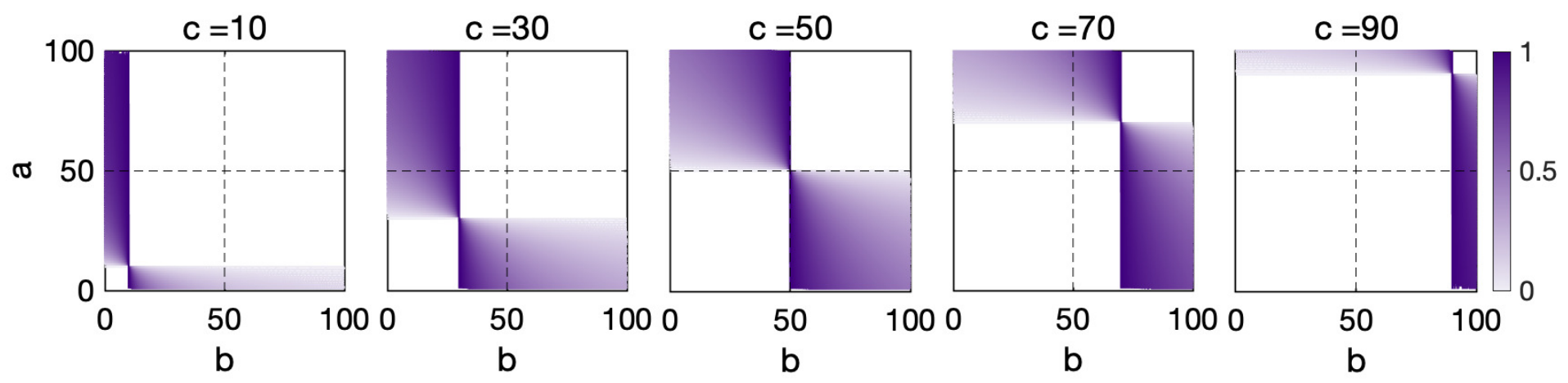

Figure 4: An analogy example of the de-mixing process with numbers. $a$ and $b$ are numbers taken from 0 to 100. $c$ is the weighted average of $a$ and $b$, given by $c=w \times a+(1-w) \times b$ (Eq. 2 ). For a given mixed number $c$, the de-mixing process is to constrain the range of possible values of $a$ and $b$ that generate $c$.

Response probabilities. Under the EP model, we aim to predict the probability that the observer reports "same" given a combination of $s_{\mathrm{p}}$ and $s_{\text {mix }}, p(\hat{C}=$ $\left.1 \mid s_{\mathrm{p}}, s_{\text {mix }}\right)$. Since $s_{\text {mix }}$ is the weighted circular mean of $s_{\mathrm{t}}$ and $s_{\mathrm{d}}$, we rewrite the response probability in terms of $s_{\mathrm{p}}, s_{\mathrm{t}}$ and $s_{\mathrm{d}}, p\left(\hat{C}=1 \mid s_{\mathrm{p}}, s_{\mathrm{t}}, s_{\mathrm{d}}\right)$ instead (see Eq.6). Again, on a single trial, the measurements of $s_{\mathrm{p}}, s_{\mathrm{d}}$ and $s_{\mathrm{t}}$ are unknown to the experimenter. Thus, we need to average over all the possible measurements $x_{\mathrm{p}}, x_{\mathrm{d}}$ and $x_{\mathrm{t}}$ to calculate the response probabilities. Therefore, the probability of reporting "same" for a given combination of $s_{\mathrm{p}}, s_{\mathrm{t}}$ and $s_{\mathrm{d}}$ is given by:

$$
\begin{aligned}
p\left(\hat{C}=1 \mid s_{\mathrm{p}}, s_{\mathrm{t}}, s_{\mathrm{d}}\right) & =\iiint p\left(\hat{C}=1 \mid x_{\mathrm{p}}, x_{\mathrm{t}}, x_{\mathrm{d}}\right) p\left(x_{\mathrm{p}} \mid s_{\mathrm{p}}\right) p\left(x_{\mathrm{t}} \mid s_{\mathrm{t}}\right) p\left(x_{\mathrm{d}} \mid s_{\mathrm{d}}\right) d x_{\mathrm{p}} d x_{\mathrm{t}} d x_{\mathrm{d}} \\
& =\iiint_{\left(x_{\mathrm{p}}, x_{\mathrm{d}}, x_{\mathrm{t}}\right): d_{\mathrm{EP}}>0} p\left(x_{\mathrm{p}} \mid s_{\mathrm{p}}\right) p\left(x_{\mathrm{t}} \mid s_{\mathrm{t}}\right) p\left(x_{\mathrm{d}} \mid s_{\mathrm{d}}\right) d x_{\mathrm{p}} d x_{\mathrm{t}} d x_{\mathrm{d}}
\end{aligned}
$$

where $d_{\mathrm{EP}}>0$ defines an area in the three-dimensional space spanned by $x_{\mathrm{t}}, x_{\mathrm{d}}$ and $x_{\mathrm{p}}$. As in NI model, we evaluated the above integral numerically on grid. The result turns out to depend on the differences of $\Delta_{\mathrm{t}} \equiv s_{\mathrm{p}}-s_{\mathrm{t}}$ and $\Delta_{\mathrm{d}} \equiv s_{\mathrm{p}}-s_{\mathrm{d}}$, but not on $s_{\mathrm{p}}, s_{\mathrm{t}}$ and $s_{\mathrm{d}}$ individually. Therefore, we will write $p\left(\hat{C}=1 \mid \Delta_{\mathrm{t}}, \Delta_{\mathrm{d}}\right)$ instead of $p\left(\hat{C}=1 \mid s_{\mathrm{p}}, s_{\mathrm{t}}, s_{\mathrm{d}}\right)$. 


\section{Late pooling model}

In addition to the EP model, we also proposed a Late Pooling (LP) model which asserts that mixing of the target and the distractor occurs at a late stage in the processing. Specifically, the observer employs a suboptimal decision rule by mixing the decision variables based on the target and distractor. Thus, on a single trial, the observer computes the decision variables based on the measurements $\left[x_{\mathrm{p}}, x_{\mathrm{d}}\right]$, $p\left(C=1 \mid x_{\mathrm{p}}, x_{\mathrm{t}}\right)$ and $\left[x_{\mathrm{p}}, x_{\mathrm{t}}\right], p\left(C=1 \mid x_{\mathrm{p}}, x_{\mathrm{t}}\right)$ separately, and then makes the final decision by taking the weighted average of the two decision variables:

$$
\begin{aligned}
d_{\mathrm{LP}} & =w d_{\mathrm{NI}}\left(x_{\mathrm{p}}, x_{\mathrm{t}}\right)+(1-w) d_{\mathrm{NI}}\left(x_{\mathrm{p}}, x_{\mathrm{d}}\right) \\
& =w \log \frac{p\left(C=1 \mid x_{\mathrm{p}}, x_{\mathrm{t}}\right)}{p\left(C=0 \mid x_{\mathrm{p}}, x_{\mathrm{t}}\right)}+(1-w) \log \frac{p\left(C=1 \mid x_{\mathrm{p}}, x_{\mathrm{d}}\right)}{p\left(C=0 \mid x_{\mathrm{p}}, x_{\mathrm{d}}\right)} \\
& =\log \frac{p(C=1)}{p(C=0)}+w \log \frac{p\left(x_{\mathrm{p}}, x_{\mathrm{t}} \mid C=1\right)}{p\left(x_{\mathrm{p}}, x_{\mathrm{t}} \mid C=0\right)}+(1-w) \log \frac{p\left(x_{\mathrm{p}}, x_{\mathrm{d}} \mid C=1\right)}{p\left(x_{\mathrm{p}}, x_{\mathrm{d}} \mid C=0\right)}
\end{aligned}
$$

The observer reports "same" when the weighted average of the decision variables $d_{\mathrm{LP}}>0$.

Response probabilities. As experimenters, we aim to predict the probability that the observer reports "same" given a stimuli combination of $s_{\mathrm{p}}, s_{\mathrm{t}}$ and $s_{\mathrm{d}}, p(\hat{C}=$ $\left.1 \mid s_{\mathrm{p}}, s_{\mathrm{t}}, s_{\mathrm{d}}\right)$. Since the corresponding measurements $x_{\mathrm{p}}, x_{\mathrm{t}}$ and $x_{\mathrm{d}}$ are all unknown to the experimenter, the experimenter needs to average over all possible combinations of $x_{\mathrm{p}}, x_{\mathrm{t}}$ and $x_{\mathrm{d}}$ to calculate the response probabilities:

$$
\begin{aligned}
p\left(\hat{C}=1 \mid s_{\mathrm{p}}, s_{\mathrm{t}}, s_{\mathrm{d}}\right) & =\iiint p\left(\hat{C}=1 \mid x_{\mathrm{p}}, x_{\mathrm{t}}, x_{\mathrm{d}}\right) p\left(x_{\mathrm{p}} \mid s_{\mathrm{p}}\right) p\left(x_{\mathrm{t}} \mid s_{\mathrm{t}}\right) p\left(x_{\mathrm{d}} \mid s_{\mathrm{d}}\right) d x_{\mathrm{p}} d x_{\mathrm{t}} d x_{\mathrm{d}} \\
& =\iiint_{\left(x_{\mathrm{p}}, x_{\mathrm{d}}, x_{\mathrm{t}}\right): d_{\mathrm{LP}}>0} p\left(x_{\mathrm{p}} \mid s_{\mathrm{p}}\right) p\left(x_{\mathrm{t}} \mid s_{\mathrm{t}}\right) p\left(x_{\mathrm{d}} \mid s_{\mathrm{d}}\right) d x_{\mathrm{p}} d x_{\mathrm{t}} d x_{\mathrm{d}}
\end{aligned}
$$

We evaluated the integral numerically on grid. The results again depends only on the difference of $\Delta_{\mathrm{t}} \equiv s_{\mathrm{p}}-s_{\mathrm{t}}$ and $\Delta_{\mathrm{d}} \equiv s_{\mathrm{p}}-s_{\mathrm{d}}$ but not on $s_{\mathrm{p}}, s_{\mathrm{t}}$ and $s_{\mathrm{d}}$ individually. Thus, we will re-write the decision variable in terms of $\Delta_{\mathrm{t}}$ and $\Delta_{\mathrm{d}}$ instead: $p\left(\hat{C}=1 \mid \Delta_{\mathrm{t}}, \Delta_{\mathrm{d}}\right)$.

\section{Substitution model}

Different from the pooling models, the Substitution (Sub) model asserts that the observer swaps the target for the distractor on some proportion of trials because of them losing track of which one is the target and which one is the distractor. 
Consequently, the observer makes the decision by choosing the $C$ with the highest posterior probability that is computed based on either the measurements of $s_{\mathrm{p}}$ and $s_{\mathrm{t}}, p\left(C=1 \mid x_{\mathrm{p}}, x_{\mathrm{t}}\right)$ or the measurements of $s_{\mathrm{p}}$ and $s_{\mathrm{d}}, p\left(C=1 \mid x_{\mathrm{p}}, x_{\mathrm{d}}\right)$, on a single trial. We define the decision variable on a single trial either as the log posterior probability ratio based on $x_{\mathrm{p}}$ and $x_{\mathrm{t}}$ :

$$
\begin{aligned}
d_{\mathrm{NI}}\left(x_{\mathrm{p}}, x_{\mathrm{t}}\right) & =\log \frac{p\left(C=1 \mid x_{\mathrm{p}}, x_{\mathrm{t}}\right)}{p\left(C=0 \mid x_{\mathrm{p}}, x_{\mathrm{t}}\right)} \\
& =\log \frac{p(C=1)}{p(C=0)}+\log \frac{p\left(x_{\mathrm{p}}, x_{\mathrm{t}} \mid C=1\right)}{p\left(x_{\mathrm{p}}, x_{\mathrm{t}} \mid C=0\right)}
\end{aligned}
$$

or the log posterior probability ratio based on $x_{\mathrm{p}}$ and $x_{\mathrm{d}}$ :

$$
\begin{aligned}
d_{\mathrm{NI}}\left(x_{\mathrm{p}}, x_{\mathrm{d}}\right) & =\log \frac{p\left(C=1 \mid x_{\mathrm{p}}, x_{\mathrm{d}}\right)}{p\left(C=0 \mid x_{\mathrm{p}}, x_{\mathrm{d}}\right)} \\
& =\log \frac{p(C=1)}{p(C=0)}+\log \frac{p\left(x_{\mathrm{p}}, x_{\mathrm{d}} \mid C=1\right)}{p\left(x_{\mathrm{p}}, x_{\mathrm{d}} \mid C=0\right)}
\end{aligned}
$$

The observer responds "same" when $d_{\mathrm{NI}}\left(x_{\mathrm{p}}, x_{\mathrm{t}}\right)>0$ or $d_{\mathrm{NI}}\left(x_{\mathrm{p}}, x_{\mathrm{d}}\right)>0$.

Response probabilities. We predict the probability of observers reporting "same". Due to the fact that the observer confuses the target with the distractor, we need to first compute the response probability that the observer reports "same" as a function of $s_{\mathrm{p}}$ and $s_{\mathrm{t}}, p\left(\hat{C}=1 \mid s_{\mathrm{p}}, s_{\mathrm{t}}\right)$ and of $s_{\mathrm{p}}$ and $s_{\mathrm{d}}, p\left(\hat{C}=1 \mid s_{\mathrm{p}}, s_{\mathrm{d}}\right)$ separately. The resulting response probability is a weighted average of the two, given by:

$$
p\left(\hat{C}=1 \mid s_{\mathrm{p}}, s_{\mathrm{d}}, s_{\mathrm{t}}\right)=w p\left(\hat{C}=1 \mid s_{\mathrm{p}}, s_{\mathrm{t}}\right)+(1-w) p\left(\hat{C}=1 \mid s_{\mathrm{p}}, s_{\mathrm{d}}\right)
$$

where $w$ and $1-w$ here denote the probability of reporting "same" based on $\left[s_{\mathrm{p}}, s_{\mathrm{t}}\right]$ and $\left[s_{\mathrm{p}}, s_{\mathrm{d}}\right]$, respectively. Method of calculating $p\left(\hat{C}=1 \mid s_{\mathrm{p}}, s_{\mathrm{t}}\right)$ and $p\left(\hat{C}=1 \mid s_{\mathrm{p}}, s_{\mathrm{d}}\right)$ is the same as in the NI model (see Eq.2 ). As indicated in Eq.2, the final response probability depends only on $\Delta_{\mathrm{t}} \equiv s_{\mathrm{p}}-s_{\mathrm{t}}$ and $\Delta_{\mathrm{d}} \equiv s_{\mathrm{p}}-s_{\mathrm{d}}$, not on $s_{\mathrm{p}}, s_{\mathrm{d}}$ and $s_{\mathrm{t}}$ individually.

\section{Comparison of decision variables}

To distinguish NI, EP and LP models, we compared the decision variable for each of the three models (see Fig.5). (The decision variable of Sub model is identical to that of the NI model and thus is not shown here.)

Under the NI model, the observer's decision of reporting "same" or "different" is determined solely by $\Delta_{x \mathrm{t}}$ (Eq.1). It predicts that the observer reports "same" when 
$\Delta_{x \mathrm{t}}$ lies within a small range (due to noisy measurements) that is centered around $0^{\circ}$. Under the EP model, the observer's decision of reporting "same" depends on $x_{\mathrm{p}}$ and $x_{\text {mix }}$ (Eq.4). Since $x_{\text {mix }}$ is the weighted circular mean of $x_{\mathrm{t}}$ and $x_{\mathrm{d}}$, we can rewrite the decision variable of EP model in terms of $\Delta_{x \mathrm{t}} \equiv x_{\mathrm{p}}-x_{\mathrm{t}}$ and $\Delta_{x \mathrm{~d}} \equiv x_{\mathrm{p}}-x_{\mathrm{d}}$ (Eq.6). Consequently, the observer's decision depends on both $\Delta_{x \mathrm{t}}$ and $\Delta_{x \mathrm{~d}}$ and varies for different $w$. When $w<0.5$ (e.g., $w=0.25$ ), the observer's decision variable equals 0 . In this case, the observer's decision is subjective to random guess because the probability of reporting "same" is 0.5 . When $w \geq 0.5$, the observer's decision variable is shaped by both $\Delta_{x \mathrm{t}}$ and $\Delta_{x \mathrm{~d}}$. Specifically, as $w$ increases over 0.5 , the influence from $\Delta_{\mathrm{d}}$ becomes smaller and diminishes to null when $w=1$. Under the LP model, by contrast, the observer's decision variable is the weighted average of the decision variables based on $\Delta_{x \mathrm{t}}$ and $\Delta_{x \mathrm{~d}}$ separately. Thus, the observer's response is largely determined by $\Delta_{x \mathrm{~d}}$ when $w$ is small but gradually dominated by $\Delta_{x \mathrm{t}}$ as $w$ is approaching 1. 


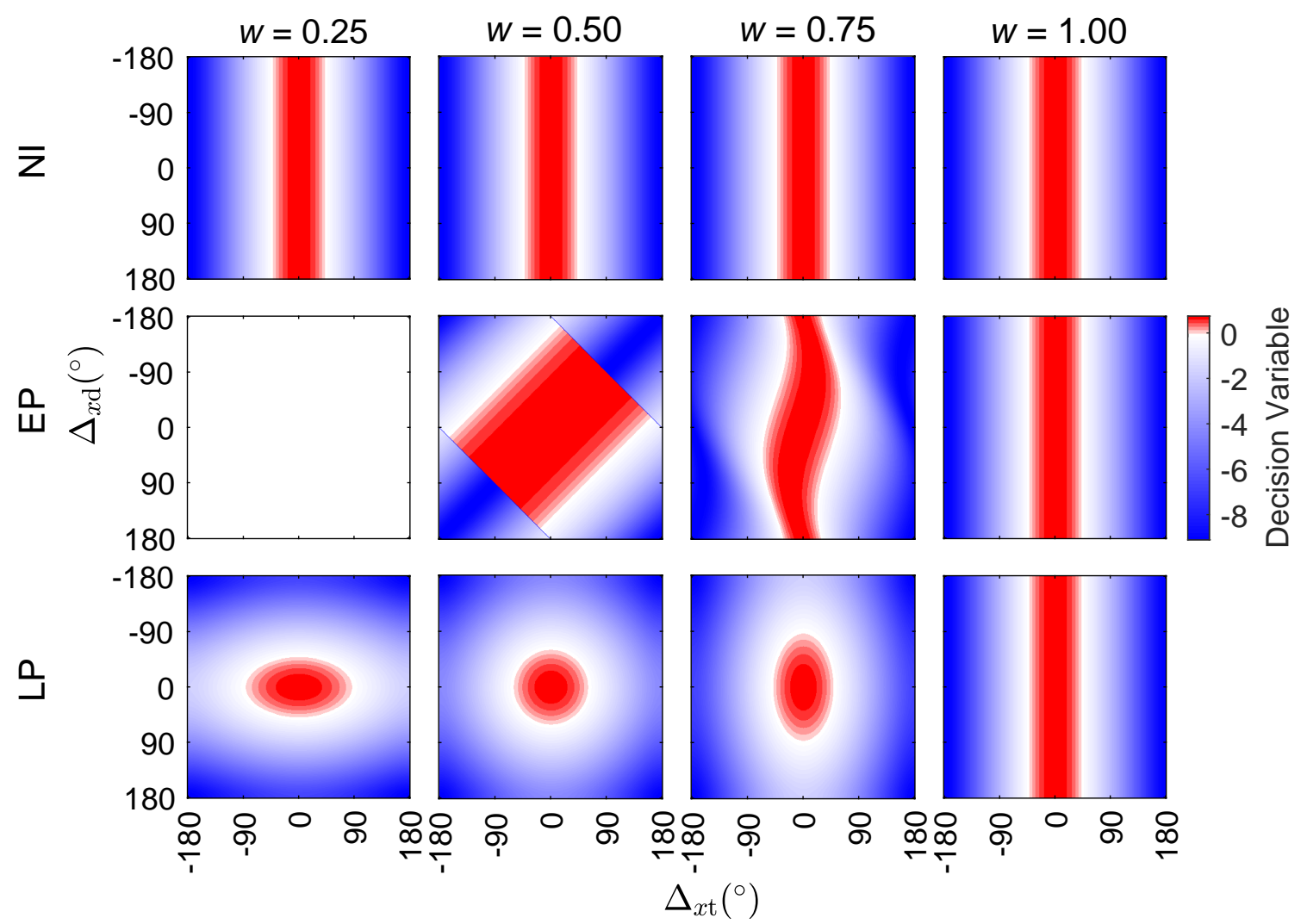

Figure 5: Decision variables for NI, EP and LP models. Decision variable depends solely on $\Delta_{x \mathrm{t}}$ in NI model, but jointly on $\Delta_{x \mathrm{t}}$ and $\Delta_{x \mathrm{~d}}$ in EP and LP models. $w$ indicates the weight for $\Delta_{x \mathrm{t}}$. The observer reports "same" when the decision variable is greater than 0 and guesses randomly when it equals 0 .

\section{Model fitting and model comparison}

The NI model has three free parameters: a shared concentration parameter $\kappa$ for the target and probe, a lapse rate $\lambda$ and a prior parameter $p(C=1)$ in the decision variable. The three interference models have one additional weighting parameter $w$. In addition to the simplified version of the four models that assume a shared $\kappa$ for the probe, distractor and target, we fitted the data with the full version of each of the four models. The full models assert different concentration parameters for the target $\kappa_{\mathrm{t}}$, distractor $\kappa_{\mathrm{d}}$ and probe $\kappa_{\mathrm{p}}$. Thus, with the full version, the NI model has two less parameters $\left(\kappa_{\mathrm{d}}\right.$ and $\left.w\right)$ than each of the three interference models. The derivation of the full version of each model is largely the same as the simplified version (see 
Appendix A for details).

We optimized the log likelihoods of the models using Bayesian adaptive direct search (BADS; Acerbi and Ma, 2017). BADS alternates between a series of fast, local Bayesian optimization steps and a systematic, slower exploration of a mesh grid. We used Akaike information criterion (AIC) and Bayesian information criterion(BIC) to compare model fits between the interference model and non-interference models. In the present study, we compared the sums of the AIC/BIC difference between each pair of the four models.

\section{Psychophysical experiment}

\section{Rationale}

In the experiment, we aimed to measure interference in the Parametric Two-Back task in which the stimuli varied parametrically on one-dimensional continua, allowing us to measure complete psychometric curves. In addition, we introduced an important control condition, in which the stimulus feature alternated between orientation and color within a stimulus sequence, thereby minimizing the potential of the 1-back item to interfere.

\section{Experimental methods}

\section{Apparatus and stimuli}

The stimuli were displayed on an iPad Retina screen with a resolution of $1280 \times 960$ pixels and a refresh rate of $60 \mathrm{~Hz}$. The screen was attached to an adjustable arm that was mounted on a rail. A chin rest was mounted on the same rail. Subjects were seated in a dark room and viewed the display from a distance of $40 \mathrm{~cm}$, so that $1 \mathrm{~cm}$ on the screen corresponded to approximately 1.4 degree of visual angle (dva).

All stimuli were generated with Psychophysics Toolbox 3 in MATLAB (Brainard, 1997; Pelli, 1997). The background was medium gray, with a luminance of around $39 \mathrm{~cd} / \mathrm{m}^{2}$. The fixation cross was white with a luminance of around $195 \mathrm{~cd} / \mathrm{m}^{2}$, and consisted of a horizontal and a vertical line segment, each of length 0.33 dva. An orientation stimulus was an oriented ellipse with a long axis of 2.9 dva, a short axis of $1.7 \mathrm{dva}$, and a luminance of around $120 \mathrm{~cd} / \mathrm{m}^{2}$. The orientation of an ellipse was randomly selected from a discrete uniform distribution on the integers between $0^{\circ}$ and $179^{\circ}$. A color stimulus was a colored disc with a diameter of 2.9 dva and a color drawn from a discrete uniform distribution on 360 color values (luminance: around 
$\left.40 \mathrm{~cd} / \mathrm{m}^{2}\right)$ that were evenly distributed along a circle in the fixed- $L$ plane of CIE $1976\left(L^{*}, a^{*}, b^{*}\right)$ color space $(L=54$ center $a=18, b=8)$ and radius 59 . The center point of the fixation point, the ellipse, or the disc was at the center of the screen.

\section{Trial}

An Orientation-Only (or Color-Only) sequence began with the appearance of the fixation cross for $1000 \mathrm{~ms}$, followed by a sequence of oriented ellipses (or colored disc). Each ellipse (disc) was presented for $800 \mathrm{~ms}$, followed by an inter-stimulus interval (ISI) of $1000 \mathrm{~ms}$, during which the subject had to respond whether the stimulus they just saw (probe) had the same orientation (or color) as the stimulus that was presented 2 stimuli earlier (target) in the sequence. The subject pressed $J$ for "same" and F for "different". If the subject did not respond within the $1000 \mathrm{~ms}$ ISI, the trial was counted as a "time-out". After the subject's response or after the timeout, auditory feedback was given, indicating a correct response (high-pitched sound), an incorrect response (low-pitched sound), or a time-out (buzz sound) (see Fig.6). Trial procedure was the same for Alternating sequence except that the fixation cross was followed by a sequence of alternating ellipses and discs. Thus, the subject had to alternate between comparing the orientation of an ellipse (probe) to the previous oriented ellipse (target, still 2 stimuli earlier in the sequence) and comparing the color of a disc (probe) to the previous colored disc (target).

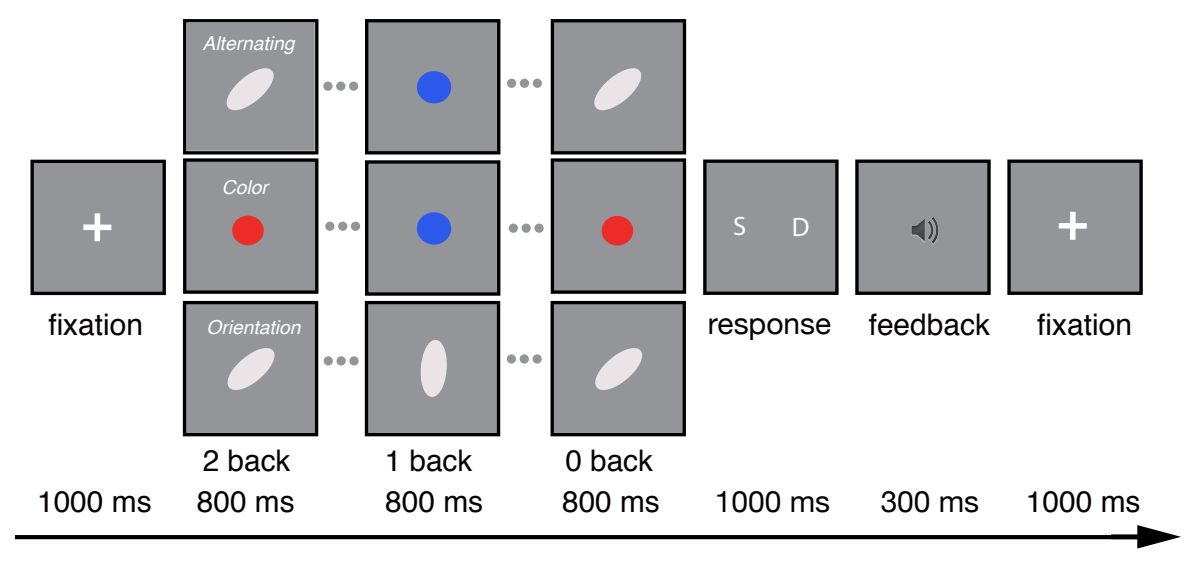

Figure 6: Trial procedure of Alternating sequence, Color-Only sequence, and OrientationOnly sequence. Each trial starts with a fixation cross, followed by one of the three sequences. After reporting the "same" or "different", the subject receives an auditory feedback, indicating a correct response (high-pitched sound), an incorrect response (low-pitched sound), or a time-out (buzz sound) 


\section{Sequence}

An Orientation-Only (Color-Only) sequence consisted of 53 items and an Alternating sequence consisted of 56 items. The first two items in each sequence was removed from analysis because they had no 2-back stimuli. Thus, there were 51 valid items for an Orientation-Only (Color-Only) sequence and 54 valid items for an Alternating sequence. An Alternating sequence consisted of 27 oriented ellipses and 27 colored discs. Sequence lengths were chosen to ensure that the orientation or color of the probe was the same as that of the target on exact $1 / 3$ of trials and was different on the remaining two thirds of trials in a sequence.

The three sequence types gave rise to four conditions (trial types): an orientation trial in an Orientation-Only sequence (Ori), a color trial in a Color-Only sequence $(\mathrm{Col})$, an orientation trial in an Alternating sequence (Ori-A), and a color trial in an Alternating sequence (Col-A). In the Ori-A and Col-A conditions, the 1-back stimulus did not possess the same feature as the probe, thus serving as control conditions to the Ori and Col conditions, respectively. We expected interference in Ori-A and Col-A condtions to be minimal.

\section{Experiment procedure}

The entire experiment consisted of 16 Orientation-Only sequences, 16 Color-Only sequences, and 32 Alternating sequences, resulting in a total of 64 sequences. The sequences were grouped into blocks, each of which consisted of 4 sequences of the same type. Consequently, the experiment was composed of 4 Orientation-Only blocks, 4 Color-Only blocks, and 8 Alternating blocks. The 16 blocks were divided over 4 sessions of 4 blocks each. In the first session, each subject performed one block of each of the three types, with the type of the fourth block randomly chosen. The remaining 12 blocks were divided over the remaining 3 sessions. Within each session, the order of the blocks was randomized. Overall, the subject completed 848 Ori trials, 848 Col trials, 896 Ori-A trials, and 896 Col-A trials.

In the first session, at the start of each new block type, the subject practiced a 20-item sequence in that block in the presence of the experimenter. Thus, the subject experienced three separate practice sequences in the first session. No practice was given in later sessions.

\section{Subjects}

Nine subjects (five female, four male, average age: 21 years old) participated. All subjects had normal or corrected-to-normal vision. Each subject completed four 
1-hour sessions and was compensated at $\$ 10$ per session, with a $\$ 10$ completion bonus. The study adhered to the Declaration of Helsinki and was approved by the institutional Review Board of New York University.

\section{Experimental results}

We first reported subjects' overall performance (Fig. 7). Planned t-tests showed that the overall accuracy was significantly higher in Ori-A condition $(0.799 \pm 0.024($ mean $\pm \mathrm{SEM}))$ than in Ori condition $[0.730 \pm 0.021, t(8)=4.59, p=0.0018]$, and was also significantly higher in Col-A condition $(0.863 \pm 0.012)$ than in Col condition $[0.815 \pm$ $0.022, t(8)=2.88, p=0.021]$. To further examine subjects' performance across the four conditions, we broke down the overall accuracy into hit rate and correct rejection rate. Planned t-tests on the rate of correct rejection showed that while there was no significant difference between the Col $(0.865 \pm 0.019)$ and Col-A $(0.879 \pm 0.013)$ conditions $[t(8)=1.02, p=0.34]$, it was significantly higher in Ori-A condition $(0.839 \pm 0.019)$ than in Ori condition $[0.782 \pm 0.019, t(8)=4.06, p=0.0036]$. In addition, the hit rate was significantly higher in Col-A condition $(0.829 \pm 0.030)$ than in Col condition $[0.715 \pm 0.043, t(8)=3.88, p=0.0047]$, but not significantly different between Ori $(0.625 \pm 0.054)$ and Ori-A $(0.715 \pm 0.078)$ conditions $[t(8)=2.04, p=0.075]$. Together, the results suggest that the 1-back distractor induced larger interference in Ori and Col conditions than in Ori-A and Col-A conditions, leading to an overall lower performance. 


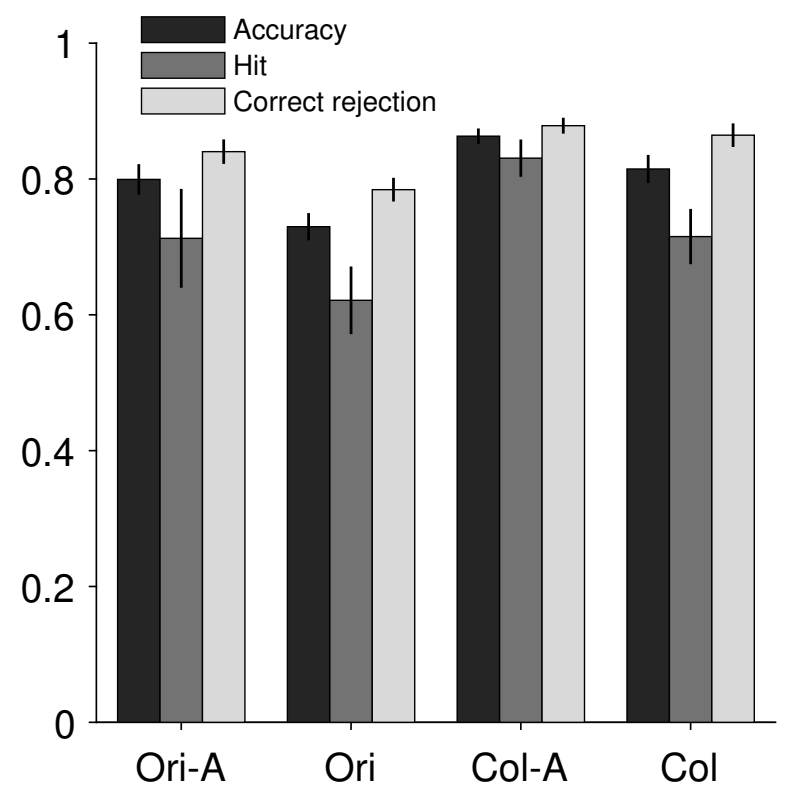

Figure 7: Overall accuracy, hit rate on reporting "same", and correct rejection rate on reporting "different" in the four conditions. Error bars indicate \pm 1 standard errors of the means.

We then examined the probability of reporting "same" as a function of $\Delta_{\mathrm{t}}$ and/or $\Delta_{\mathrm{d}}$ in the four conditions (Fig. 8). The probability of reporting "same" peaked when $\Delta_{\mathrm{t}}$ was $0^{\circ}$ and decreased with an increase in $\Delta_{\mathrm{t}}$. Subjects also reported more false alarms in Ori and Col conditions than in Ori-A and Col-A conditions (Fig. 8A and $\mathrm{F}$ ), indicating larger interference in the former two conditions where features of the target and distractor were from the same category. In addition, the strength of interference was also dependent on $\Delta_{d}$ : the probability of reporting "same" was overall higher when $\Delta_{\mathrm{d}}$ was small (e.g., in the range of $0^{\circ}-15^{\circ}$ for orientation and $0^{\circ}-30^{\circ}$ for color), indicating larger interference when features of the distractor and probe were close (i.e., $\Delta_{\mathrm{d}}$ is around $0^{\circ}$ ) (Fig.8B and $\mathrm{G}$ ).

We further characterized the distractor dependence of interference by computing the probability of reporting "same" as a function of $\Delta_{\mathrm{d}}$ with different bins of $\Delta_{\mathrm{t}}$ (Fig. 8C, D, H, and I). Again, the probability of reporting "same" was highest when $\Delta_{\mathrm{d}}$ was small and flattened out as $\Delta_{\mathrm{d}}$ continued to increase, suggesting that the distractor induced negligible interference when its value was away from the probe (Fig. 8C and H). This pattern persisted for different bins of $\Delta_{\mathrm{t}}$ (Fig. 8D and I). The joint effect of $\Delta_{\mathrm{t}}$ and $\Delta_{\mathrm{d}}$ on responses was dispalyed in Fig. 8E and J. The highest 
probability of reporting "same" lied in a region where both $\Delta_{\mathrm{t}}$ and $\Delta_{\mathrm{t}}$ were around $0^{\circ}$.

To further verify the effect of the 1-back distractor on subjects' performance, we fitted 2-variable logistic regressions (with $\left|\Delta_{t}\right|$ and $\left|\Delta_{d}\right|$ as two regressors) to each individual's data for both Ori and Col conditions. One-sample t-tests showed that both coefficients were significantly different from 0 in Ori condition $[-0.045 \pm$ $0.0083($ mean \pm SEM $), t(8)=-5.78, p=0.00041$ for $\left|\Delta_{\mathrm{t}}\right|$ and $-0.0071 \pm 0.0022, t(8)=$ $-3.52, p=0.0078$ for $\left.\left|\Delta_{\mathrm{d}}\right|\right]$ and Col condition $[-0.036 \pm 0.0047, t(8)=-8.02, p<$ $10^{-4}$ for $\left|\Delta_{\mathrm{t}}\right|$ and $-0.0027 \pm 0.00085, t(8)=-5.78, p=0.0088$ for $\left.\left|\Delta_{\mathrm{d}}\right|\right]$, indicating that the 1-back distractor indeed affacted human performance.

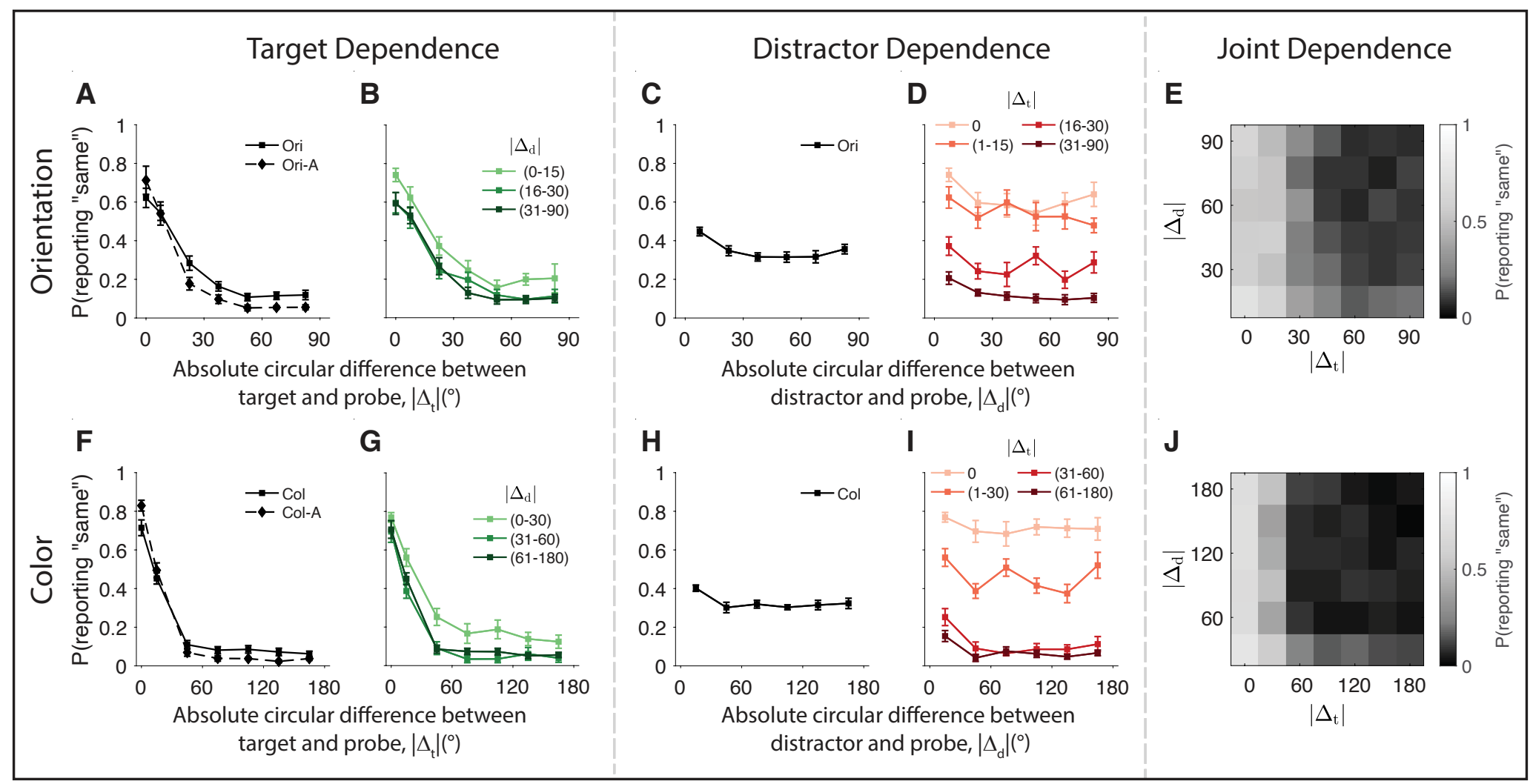

Figure 8: Behavioral data. (A) Average proportion of reporting "same" as a function of $\Delta_{t}$ in the Ori and Ori-A conditions, and (B) for different bins of $\Delta_{\mathrm{d}}$ in the Ori condition. (C) Average proportion of reporting "same" as a function of $\Delta_{\mathrm{d}}$ in the Ori condition and (D) for different bins of $\Delta_{t}$. (E) Proportion of reporting "same" as a function of $\Delta_{t}$ and $\Delta_{d}$. Error bars indicate one standard error below and above the mean. (F-J) The equivalents of B-F for the stimulus of Color. 


\section{Modeling results}

We fitted behavioral data from Ori and Ori-A conditions jointly and from Col and Col-A conditions jointly with both the simplified and the completed versions of each model. Each subject's data were fitted individually. Since introducing different precision parameters for the target, the distractor, and the probe did not improve the fitting much, in the following section we only reported model fits from the simplified version of all four models.

Average model fits to both the orientation and color conditions were displayed in Fig 9. Consistent with behavioral data, all four models predicted that the probablity of reporting "same" reduced as a function of $\Delta_{t}$ (Fig.9B - E). However, the four models behaved differently in predicting the strength of interference from the 1-back distractor. It was no surprise that the NI model predicted no inteference from the distractor. The EP model that assumes a mixing of the target and distractor features did not capture the effect of $\Delta_{d}$ either. In contrast, both the LP and Sub models predicted an overall higher probability of reporting "same" when $\Delta_{\mathrm{d}}$ was small (e.g., $\Delta_{\mathrm{d}}$ was within a range of $\left[0^{\circ} 15^{\circ}\right]$ for orientation and $\left[0^{\circ} 30^{\circ}\right]$ for color. See Fig $9 \mathrm{~B}$, $\mathrm{C}, \mathrm{E}$, and $\mathrm{F}$ ). These two models also captured well the joint effect of $\Delta_{\mathrm{t}}$ and $\Delta_{\mathrm{d}}$ on the peformance (Fig.9 A and D).

While the LP and Sub models outperformed the NI and EP models, they fitted the data comparably well and appeared to be indistinguishable in both the orientation and color conditions. Fitted parameter estimates for each of the four models are displayed in Appendix D (Tables S1 and S2). 


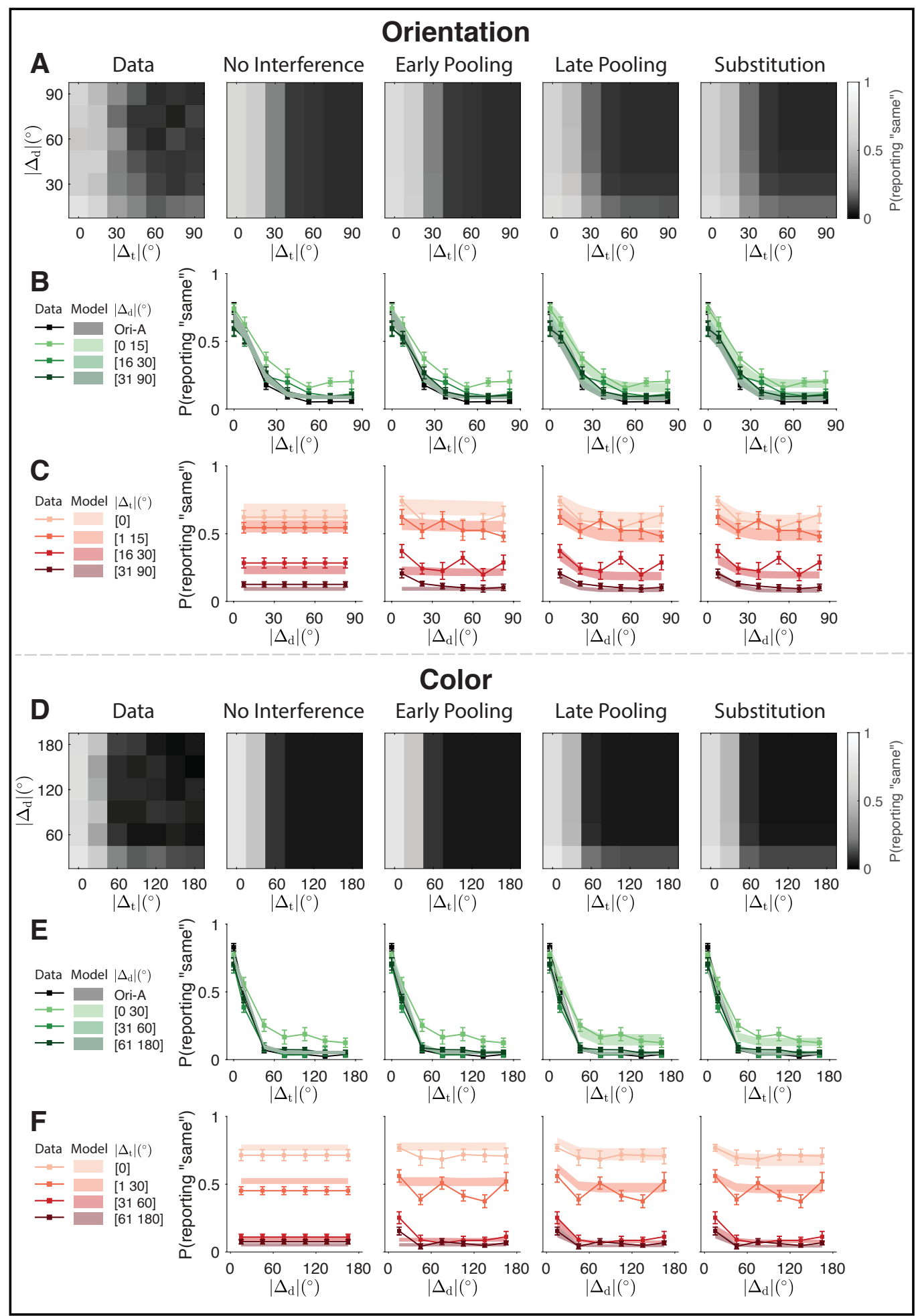

Figure 9: Model fits. Panel A: Proportion of reporting "same" as a function of $\Delta_{t}$ and $\Delta_{d}$ for observed data (column 1) and each of the model predictions (columns 2 to 5). Panel B: Proportion of reporting "same" as a function of $\Delta_{t}$ for different bins of $\Delta_{d}$. Panel C: Proportion of reporting "same" as a function of $\Delta_{\mathrm{d}}$ for different bins of $\Delta_{\mathrm{t}}$. Discrete points indicate observed data points and shaded colored areas indicate model fits. Error bars indicate one standard error above and below the means of observed data points. Panels D-E show color condition and correspond to panels A-C in orientation condition, respectively. 
To compare model performance quantitatively, we calculated the summed difference in AIC and BIC between each pair of the four models (see Appendix E for detailed model comparsion results). 95\% confidence intervals of their summed difference were calculated from 5000 bootstrapped samples.

The sums of AIC difference between NI model and EP model, LP model, Sub model were $-58([-130,-18]),-204([-441,-76])$ and $-252([-446,-115])$, respectively for orientation and were $-74([-117,-30]),-218([-367,-97])$, and $-238([-433,-100])$, respectively for color (Fig.10). The negative summed differences indicate that NI model performed the worst among the four models for both orientation and color stimuli. Among the three interference models, the sum of AIC difference was -148([-363, -45]) (for orientation) and -144([-268, -55]) (for color) between EP model and LP model and was -197([-344, -89]) (for orientation) and -164([-328, -58]) (for color) between EP model and Sub model, revealing better performances for LP and Sub models than EP model. The summed difference between SuP model and LP model was $-49([-114,1.3])$ for orientation and $-20([-66,5.9])$ for color, indicating that Sub model was slightly better than LP model.

Model Comparison based on BIC revealed a similar pattern except for a smaller advantage of EP model over NI model (Fig.10). Specifically, the sum of BIC difference between the NI model and EP model was -13([-91, 24]) for orientation and $-31([-74,13])$ for color, indicating that EP model was only slightly better than NI model. The small discrepancy between AIC and BIC difference was due to the fact that BIC introduced a larger penalty than AIC. Since NI model had one less parameter than the other three interference models, the small summed AIC difference between the NI and EP models was counteracted by a larger penalty imposed by BIC.

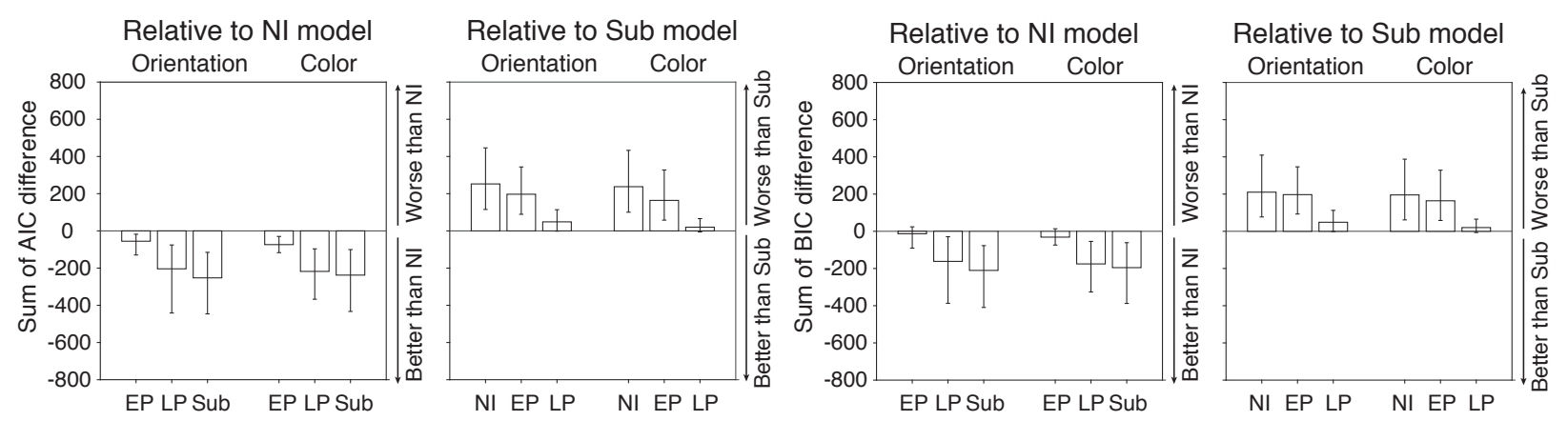

Figure 10: Model comparison. Sum of AIC and BIC relative to that of the No Interference model or Substitution model for both color and orientation across subjects. Error bars indicate $95 \%$ confidence intervals of the sums obtained from boostrapping. 


\section{Discussion}

In the present study, we aimed to quantify the magnitude of inteference induced by a 1-back distractor in the 2-back task and disentangle the underlying sources of inteference.

Short summary of experimental results. To quantitatively measure inteference, we created a new variant of the classic 2-back task by employing simple parametric stimuli (orientation and color) that were varied on a trial-by-trial basis. The Parametric Two-Back Task enabled us to measure the magnitude of 1-back-iteminduced interference as a function of distractor-probe similarity (i.e., feature distance of the probe and distractor). In the experiment, we observed larger interference when feature distance of the probe and distractor was closer. Interference was also greater in the Orientation- and Color-only conditions than in the Orientation-Color alternating condition where features of the target and distractor were qualitatively different. The results were in line with prior studies, which used categorical stimuli (e.g., letters and numbers) and found larger interference when the probe and distractor were identical than when they were different (Kane et al., 2007; Moore and Ross, 1963).

Short summary of modeling results. We proposed three hypotheses to account for the possible sources of interference induced by the 1-back distractor. Specifically, interference could arise from the observer either (1) mixing the features of the target and distractor at an early stage of the processing (i.e., perceptual stage), or (2) mixing the strengths of evidence obtained separately from the target and distractor at a late stage of the processing, or (3) confusing the identities of the target and distractor on some proportion of trials. We tested each of the three hypotheses by comparing three interference models: the Early pooling (NP) model, the Late pooling (LP) model and the Substitution (Sub) model. All three interference models are based on the No interference (NI) model that posits no interference from the 1-back distractor.

Model comparisons based on both AIC and BIC indicated that LP and Sub models outperformed NI and EP models. However, the LP model and Sub model fitted the data comparably well. Thus, our modeling results suggest that interference in the 2-back task results from the observer either mixing the strengths of evidence (represented by decision variables) based on the target and distractor separately or substituting the distractor for the target with a variable probability on a single trial.

Comparison to similar models in visual crowding. Our EP model is conceptually similar to the pooling models proposed in the crowding literature, which assume that the observer takes the average of the perceived features of the target and distractors (e.g., flankers) that are corrupted by independent additive (e.g.Gaussian) 
noise (Parkes et al., 2001; Greenwood et al., 2009). Nevertheless, our EP model differs from them on an important aspect. Specifically, the EP model is constructed from an optimal observer model, which allows it to implement an optimal de-mixing decision strategy in spite of an inefficient encoding process (i.e., mixing of the target and distractor features). The optimal decision strategy enables it to reconstruct the original target and distractor features from the mixed feature. In contrast, such a de-mixing process is absent in pooling models from the crowding literature.

To our knowledge, there are no comparable models in the crowding literature to our LP model. All pooling models in the crowding literature assert that the observer mixes the features of the target and distractor in the early stage of the processing (i.e., encoding stage) and prior to reaching awareness (Parkes et al., 2001; Greenwood et al., 2009; Ester et al., 2014, 2015). Neural population code models also implement the feature pooling hypothesis (Van den Berg et al., 2010; Harrison and Bex, 2015; Orhan and Ma, 2015). In contrast, our LP model assumes that the observer mixes the strengths of evidence from the target and the distractor separately at a late stage of the processing (i.e., decision stage). Moreover, it is worth noting that under our model framework, we are also able to implement the mixing of other aspects of the target and distractor. For instance, a possible, untested pooling model could be that the observer mixes the strengths of signal from the target and the distractor separately (i.e., the measured changes of $\Delta_{t}$ and $\Delta_{d}$, see Fig 2). Thus, our LP model considerably extends the theoretical domain of the pooling hypothesis.

Our Sub model is also conceptually inspired by similar substitution models in the crowding literature (Greenwood et al., 2009; Ester et al., 2014, 2015; Harrison and Bex, 2017), which, in general, postulate that the observer swaps the target for the distractor with a variable probability because of them losing identities of the items (i.e., confusing the target with the distractor). Our Sub model holds the same underlying assumption but implements it under the Bayesian inference framework. As a result, the observer could still employ the optimal decision strategy for the target and the distractor separately.

To summarize, while the existing pooling and substitution models in the crowding literature provide the basic assumptions that inspire our work, our models, formalized under the Bayesian inference framework, refine them on several major aspects. Firstly, our NI model offers a benchmark for model comparison, as each of the interference models differs from it in only one component. This stands in contrast with the previous models that could introduce somewhat arbitrary computation processes, rendering model comparison less satisfactory. Secondly, we derived the optimal demixing decision strategy for the EP model, which could also be used to predict performance in other cognitive tasks, such as change detection or crowding, where 
the observer may suffer from interference induced by the distracting items. Thirdly, our LP model extends the pooling hypothesis by proposing that averaging of the target and distractor could occur outside of the feature space.

Other interference models in working memory. Interference is not specific to the $N$-back task. It is, in fact, a quite common phenomenon in working memory. Studies have shown that the previously stored information could still interfere with processing of the current information, even when it becomes irrelevant to the task and is no longer needed in working memory (Jonides and Nee, 2006; Makovski and Jiang, 2008).

Interference among representations of different items has been suggested as an alternative theory to explain working memory capacity limits (Oberauer et al., 2012; Oberauer and Kliegl, 2006; Nairne, 2002). Oberauer and Lin (2017) proposed an interference model for working memory for continuous visual information. The model is characterized by a 2-layer connectionist network, a feature layer representing all the memory contents (e.g., color or orientations) and a context layer representing the (temporal or spatial) contexts at which the corresponding memory contents are encoded. A set of items is encoded by binding each to its context (i.e., a time point or a specific position at which the item appears). Bindings are formed and modified through a Hebbian learning rule. Consequently, retrieval of each item is achieved by re-activating its corresponding context first, which in turn re-evokes its associated memory content. Under this model, interference occurs in two scenarios: 1) item features are superimposed in the feature layer or 2) items are bound to the wrong contexts. The two scenarios align with the pooling and substitution hypotheses of interference proposed in our models, respectively.

Wei et al. (2012) introduced a biophysical realistic network model for working memory. The network consists of a set of spiking excitatory pyramidal cells and inhibitory interneurons (see also Compte et al., 2000). Continuous stimuli are encoded by a distributed neural population and maintained in working memory through persistent activities of distinct localized neural populations (i.e., discrete bump attractors). Under this network model, working memory capacity limit can be explained by either fading out of the local bump attractors or merging of nearby bumps. Of particular interest is the fact that the probability of merging (or pooling in our term) increases significantly as the distance between the nearby bumps decreases (i.e., as a result of increased similarity between the stimulus features) (see also Furman and Wang, 2008).

These network models provide good insights into the possible mechanisms of interference in working memory. Despite the fact our models and these network models characterize the working memory process from different levels (ranging from purely 
mathematical to biological realistic), they are in good agreement on the sources of interference: it is caused either by confusion between or pooling of multiple items.

Inteference caused by intertrial dependence. The present study tested three hypotheses regarding the possible sources of interference in the 2-back task. Nevertheless, we by no means suggest that they are the only possible sources. As performing the 2-back task involves various cognitive processes, it is very likely that interference could be induced by many other factors. One of the most prominent "other factors" we consider here is the sequential dependence across trials. Though our models assume that the trials are independent of each other, this is hardly the case in reality.

Sequential dependence has been regularly demonstrated in visual perception (Thompson and Burr, 2009; Fischer and Whitney, 2014; Fründ et al., 2014). Specifically, studies have indicated that the perceived feature of the current stimulus could be systematically biased toward (i.e., attractive effect) those of the recently seen stimuli (i.e., not confined to the immediately preceding stimulus), an indication of an underlying pooling process (i.e., averaging of the current and preceding stimuli). Related to our 2-back task, the perceived feature of the probe might be influenced by the 1-back, 2-back, or even 3-back item, which could consequently give rise to interference. Interestingly, reminiscent of our modeling goals, there is an onging debate over whether the pooling process occurs early in the perceptual stage or late in the decision stage (Fritsche et al., 2017; Fornaciai and Park, 2018; Cicchini et al., 2017). Our proposed EP and LP models could help to disentangle the two possible loci of the pooling process.

Intertrial dependence has also been reported in decision making (Abrahamyan et al., 2016; Braun et al., 2018; Vinson et al., 2019). The choice history (i.e., decision or response on the previous trial) could bias the observer's future decisions (i.e., decision on the current trial)(Abrahamyan et al., 2016; Vinson et al., 2019). For example, the observer could switch their choice on the current trial following a failed response on the past trial. In our experiment, the feedback provided after each response might create a condition for the participants to change their current decision upon being informed about the outcome of their previous one. These additional possible sources of interference, though not the focus of the current study, deserve further examination.

Limitations. One possible reason LP model and Sub model were not distinguishable was that we observed substantial interference only when the distractor-probe similarity was high. When the feature similarity is high, mathematically it is not easy to distinguish the pooling model from the substitution model because both models generate similar results (e.g., the average of $48^{\circ}$ and $52^{\circ}$ is not markedly 
different from either $48^{\circ}$ or $52^{\circ}$ ). This issue has been pointed out in the crowding literature where pooling and substitution models are difficult to tease apart when target-distractor (flanker) similarity is high (Ester et al., 2014; Hanus and Vul, 2013; Harrison and Bex, 2015). However, it is also possible that both substitution and pooling mechanisms contribute to the observed interference in our task. Studies in crowding have shown that the data can not be accounted for by either the substitution model or pooling model alone, but could be better captured by a mixture model incorporating both processes (Freeman et al., 2012; Harrison and Bex, 2017). Unfortunately, our data and modeling results do not allow us to distinguish these two possibilities.

Note that in our EP model, we did not test any suboptimal decision strategies that may simplify the decision variable for two reasons: 1) we wanted to vary only one component at a time between the models. If we had chosen a suboptimal decision strategy, then there would have been two differences between the NI and EP models, thus making it unclear what to attribute a better fit of either model; 2) while there is only one optimal strategy, there are many suboptimal decision strategies, and choosing between them is rather arbitrary and therefore harder to justify. However, we can not exclude the possibility that the observer might use some simple, though not optimal, decision strategies. One of the most straightforward sub-optimal strategies is not to perform the reconstruction (of the original target and distractor features) process. This sub-optimal strategy is appealing because of its intuition and easy implementation, and thus has been used as a default strategy in crowding models (Parkes et al., 2001; Freeman et al., 2012; Harrison and Bex, 2017; Greenwood et al., 2009; Dakin et al., 2010). It would be interesting to examine the simpler pooling models with some non-optimal decision strategies and see whether it could provide a better fit to our data.

Conclusion. In this work, we introduce the Parametric Two-Back task that enables us to characterize the magnitude of distractor-induced interference as a function of probe-distractor similarity. To probe the sources of interference, we propose two pooling models, a substitution model, and an optimal observer model. Our Early Pooling model refines the existing pooling models by assuming an optimal de-mixing decision strategy, and our Late Pooling model extends the pooling hypothesis by positing a mixture of evidences (rather than features) from the target and distractor separately. Altogether, our study provides useful experimental and modeling tools to parametrically examine the sources of interference in similar cognitive tasks. 


\section{References}

Abrahamyan, A., Silva, L. L., Dakin, S. C., Carandini, M., and Gardner, J. L. (2016). Adaptable history biases in human perceptual decisions. Proceedings of the National Academy of Sciences, 113(25):E3548-E3557.

Acerbi, L. and Ma, W. J. (2017). Practical bayesian optimization for model fitting with bayesian adaptive direct search. In Advances in Neural Information Processing Systems, pages 1834-1844.

Awh, E., Barton, B., and Vogel, E. K. (2007). Visual working memory represents a fixed number of items regardless of complexity. Psychological Science, 18(7):622628.

Bernard, J.-B. and Chung, S. T. (2011). The dependence of crowding on flanker complexity and target-flanker similarity. Journal of Vision, 11(8):1-1.

Braun, A., Urai, A. E., and Donner, T. H. (2018). Adaptive history biases result from confidence-weighted accumulation of past choices. Journal of Neuroscience, 38(10):2418-2429.

Chung, S. T. and Mansfield, J. S. (2009). Contrast polarity differences reduce crowding but do not benefit reading performance in peripheral vision. Vision Research, 49(23):2782-2789.

Cicchini, G. M., Mikellidou, K., and Burr, D. (2017). Serial dependencies act directly on perception. Journal of Vision, 17(14):1-9.

Compte, A., Brunel, N., Goldman-Rakic, P. S., and Wang, X.-J. (2000). Synaptic mechanisms and network dynamics underlying spatial working memory in a cortical network model. Cerebral Cortex, 10(9):910-923.

Conlin, J. A. and Gathercole, S. E. (2006). Lexicality and interference in working memory in children and in adults. Journal of Memory and Language, 55(3):363380.

Dakin, S. C., Cass, J., Greenwood, J. A., and Bex, P. J. (2010). Probabilistic, positional averaging predicts object-level crowding effects with letter-like stimuli. Journal of Vision, 10(10):14-14. 
Ester, E. F., Klee, D., and Awh, E. (2014). Visual crowding cannot be wholly explained by feature pooling. Journal of Experimental Psychology: Human Perception and Performance, 40(3):1022.

Ester, E. F., Zilber, E., and Serences, J. T. (2015). Substitution and pooling in visual crowding induced by similar and dissimilar distractors. Journal of Vision, 15(1):4-4.

Fischer, J. and Whitney, D. (2014). Serial dependence in visual perception. Nature Neuroscience, 17(5):738.

Fornaciai, M. and Park, J. (2018). Attractive serial dependence in the absence of an explicit task. Psychological Science, 29(3):437-446.

Freeman, J., Chakravarthi, R., and Pelli, D. G. (2012). Substitution and pooling in crowding. Attention, Perception, \& Psychophysics, 74(2):379-396.

Fritsche, M., Mostert, P., and de Lange, F. P. (2017). Opposite effects of recent history on perception and decision. Current Biology, 27(4):590-595.

Fründ, I., Wichmann, F., and Macke, J. (2014). Quantifying the effect of intertrial dependence on perceptual decisions. Journal of Vision, 14(7: 9):1-16.

Furman, M. and Wang, X.-J. (2008). Similarity effect and optimal control of multiplechoice decision making. Neuron, 60(6):1153-1168.

Greenwood, J. A., Bex, P. J., and Dakin, S. C. (2009). Positional averaging explains crowding with letter-like stimuli. Proceedings of the National Academy of Sciences, 106(31):13130-13135.

Hanus, D. and Vul, E. (2013). Quantifying error distributions in crowding. Journal of Vision, 13(4):17.

Harrison, W. J. and Bex, P. J. (2015). A unifying model of orientation crowding in peripheral vision. Current Biology, 25(24):3213-3219.

Harrison, W. J. and Bex, P. J. (2017). Visual crowding is a combination of an increase of positional uncertainty, source confusion, and featural averaging. Scientific Reports, 7:45551.

Jaeggi, S. M., Buschkuehl, M., Perrig, W. J., and Meier, B. (2010). The concurrent validity of the n-back task as a working memory measure. Memory, 18(4):394-412. 
Jonides, J. and Nee, D. E. (2006). Brain mechanisms of proactive interference in working memory. Neuroscience, 139(1):181-193.

Kane, M. J., Conway, A. R., Miura, T. K., and Colflesh, G. J. (2007). Working memory, attention control, and the n-back task: a question of construct validity. Journal of Experimental Psychology: Learning, Memory, and Cognition, 33(3):615.

Keshvari, S. and Rosenholtz, R. (2016). Pooling of continuous features provides a unifying account of crowding. Journal of Vision, 16(3):39-39.

Kirchner, W. K. (1958). Age differences in short-term retention of rapidly changing information. Journal of Experimental Psychology, 55(4):352.

Levi, D. M. (2008). Crowding - an essential bottleneck for object recognition: A mini-review. Vision Research, 48(5):635-654.

Makovski, T. and Jiang, Y. V. (2008). Proactive interference from items previously stored in visual working memory. Memory \& Cognition, 36(1):43-52.

Moore, M. E. and Ross, B. M. (1963). Context effects in running memory. Psychological Reports, 12(2):451-465.

Nairne, J. S. (2002). Remembering over the short-term: The case against the standard model. Annual Review of Psychology, 53(1):53-81.

Nandy, A. S. and Tjan, B. S. (2007). The nature of letter crowding as revealed by first-and second-order classification images. Journal of Vision, 7(2):5-5.

Oberauer, K. (2005). Binding and inhibition in working memory: individual and age differences in short-term recognition. Journal of Experimental Psychology: General, 134(3):368.

Oberauer, K., Farrell, S., Jarrold, C., Pasiecznik, K., and Greaves, M. (2012). Interference between maintenance and processing in working memory: The effect of item-distractor similarity in complex span. Journal of Experimental Psychology: Learning, Memory, and Cognition, 38(3):665.

Oberauer, K. and Kliegl, R. (2006). A formal model of capacity limits in working memory. Journal of Memory and Language, 55(4):601-626.

Oberauer, K., Lange, E., and Engle, R. W. (2004). Working memory capacity and resistance to interference. Journal of Memory and Language, 51(1):80-96. 
Oberauer, K. and Lin, H.-Y. (2017). An interference model of visual working memory. Psychological Review, 124(1):21.

Orhan, A. E. and Ma, W. J. (2015). Neural population coding of multiple stimuli. Journal of Neuroscience, 35(9):3825-3841.

Parkes, L., Lund, J., Angelucci, A., Solomon, J. A., and Morgan, M. (2001). Compulsory averaging of crowded orientation signals in human vision. Nature Neuroscience, 4(7):739.

Pochon, J., Levy, R., Fossati, P., Lehericy, S., Poline, J., Pillon, B., Le Bihan, D., and Dubois, B. (2002). The neural system that bridges reward and cognition in humans: an fmri study. Proceedings of the National Academy of Sciences, 99(8):5669-5674.

Põder, E. (2007). Effect of colour pop-out on the recognition of letters in crowding conditions. Psychological Research, 71(6):641-645.

Thompson, P. and Burr, D. (2009). Visual aftereffects. Current Biology, 19(1):R11R14.

Van den Berg, R., Roerdink, J. B., and Cornelissen, F. W. (2010). A neurophysiologically plausible population code model for feature integration explains visual crowding. PLoS Computational Biology, 6(1):e1000646.

Vinson, D. W., Dale, R., and Jones, M. N. (2019). Decision contamination in the wild: Sequential dependencies in online review ratings. Behavior Research Methods, pages $1-8$.

Wei, Z., Wang, X.-J., and Wang, D.-H. (2012). From distributed resources to limited slots in multiple-item working memory: a spiking network model with normalization. Journal of Neuroscience, 32(33):11228-11240. 